\title{
A mesoscale model for the Martian atmosphere
}

\author{
Anthony D. Toigo \\ Center for Radiophysics and Space Research, Cornell University, Ithaca, New York, USA
}

Mark I. Richardson

Division of Geological and Planetary Sciences, California Institute of Technology, Pasadena, California, USA

Received 16 March 2001; revised 30 October 2001; accepted 9 April 2002; published 12 July 2002.

[1] The Pennsylvania State University/National Center for Atmosphere Research

Mesoscale Model Version 5 (MM5) has been converted for use on Mars. Modifications are based on schemes implemented in the Geophysical Fluid Dynamics Laboratory Mars General Circulation Model (GCM). Validation of the Mars MM5 is conducted by comparison to the Mars GCM, examining the large-scale dynamics in the two models. Agreement between the two models on similar scales (a few hundred kilometers) is good. Validation is also performed against both Viking Landers and Mars Pathfinder meteorological observations with the model run at higher vertical (lowest level at $1.6 \mathrm{~m}$ ) and horizontal resolution (a few kilometers). We find reasonable agreement with nearsurface air temperature, pressure, and wind direction observations, with caveats. The results demonstrate that the model accurately simulates surface heat balance and the propagation of global thermal tides. However, wind speeds are underpredicted. The model generates the correct phasing of wind speeds with local time at the Viking Lander 2 site during winter but does not generate the correct phasing at the other sites or seasons. We examined the importance of slopes and global tides in generating the diurnal cycle of winds at the lander sites. We find that tides are at least as important as slopes, in contrast to previous studies. This study suggests that when used in combination with a GCM, the Mars MM5 promises to be a powerful tool for the investigation of processes central to the Martian climate on scales from hundreds of kilometers to tens of meters. INDEX TERMS: 3329 Meteorology and Atmospheric Dynamics: Mesoscale meteorology; 3337 Meteorology and Atmospheric Dynamics: Numerical modeling and data assimilation; 3346 Meteorology and Atmospheric Dynamics: Planetary meteorology (5445, 5739); 5445 Planetology: Solid Surface Planets: Meteorology (3346); 6225 Planetology: Solar System Objects: Mars; KEYWORDS: Mars, mesoscale, atmosphere, dynamics, model, numerical

\section{Introduction}

[2] The study of dynamical processes operating within the Martian atmosphere has benefited greatly from the modification and application to Mars of atmospheric models developed for Earth. These models have provided insight into the dynamics of the Martian general circulation, including the response of the Hadley circulation to changes in aerosol heating [Haberle et al., 1982; Wilson, 1997] and the behavior of the aerosol and volatile cycles [e.g., Pollack et al., 1993; Murphy et al., 1995; Richardson, 1999]. However, to date, these models have been global and of sufficient resolution to resolve only synoptic scale processes (greater than a few hundred kilometers). Results from global models increasingly suggest the importance of smaller-scale processes, for example, the lifting of dust from the surface and injection into the atmosphere, and the exchange of water with and transport of vapor to or from the northern polar cap. At the same time, high-resolution thermal and imaging data from the Mars

Copyright 2002 by the American Geophysical Union. 0148-0227/02/2000JE001489
Global Surveyor are now available that require atmospheric models capable of resolving motions on scales of a few hundreds of meters to a few hundreds of kilometers. These data include observations of the polar regions, dust devils, dust storms, water ice cloud systems, and aeolian features.

[3] In this paper we introduce a Martian mesoscale model that is designed to address motions on scales smaller than resolvable by current numerical models of the atmosphere. The model is based on the Pennsylvania State University (PSU)/National Center for Atmosphere Research (NCAR) Mesoscale Model Version 5 (MM5) [Dudhia, 1993] and is fully converted to Martian conditions. The model is designed to work in tandem with a global model which provides initial and boundary conditions. The mesoscale model (Mars MM5) simulates a limited domain within this global context at resolutions ranging from $10^{2}$ to $10^{5} \mathrm{~m}$. The model has been developed to address a number of outstanding problems in Martian atmospheric studies. These include the following:

- How is dust lifted from the surface and injected into the atmosphere?

- What is the nature of the polar regional circulation, and 
how does the circulation moderate transport of aerosols and volatiles into and out of the polar caps?

- What processes are important in cloud formation?

- What controls the evolution and structure of Martian dust storm systems?

- How does the atmosphere interact with the surface in terms of mechanically eroding, transporting, and depositing sediment and sculpting the surface?

- What processes control the dynamics of the boundary layer? How important are tides versus slopes in generating the diurnal cycle of wind at the surface?

[4] The application of the Mars MM5 to the problems listed above should advance the insight gained from other, more global modeling efforts. The purpose of this paper is to provide a description of the model and to compare the model to available data and to a well-tested global model. This paper will be the first in a series of papers which will use the model to investigate a wide variety of physical phenomena. The first such application will be Toigo et al. [2002]. This study is analogous to careful calibration and characterization of a particularly complex piece of experimental apparatus. Thus the current paper has two purposes. The first is to fully describe the Mars MM5 and the physical parameterizations that distinguish it from the well-documented terrestrial MM5 model. This description is provided in section 2 along with a discussion of the global model which is used to provide context. The second purpose is to demonstrate the validity of the model as compared to the global model (when operated at similar resolution) and to the available surface weather station data. The comparison to the global model is discussed in section 3, and that to the surface meteorological observations is discussed in section 4. In the latter case we demonstrate that simulations executed with resolutions of a few tens of kilometers can explain most of the diurnal variability of temperature, pressure, and winds at the landing sites. Finally, in section 5 we provide a summary.

\section{Model Descriptions}

\subsection{Mars MM5}

[5] The basis of the model used is the fifth-generation (version 3) PSU/NCAR Mesoscale Model (MM5), which we have adapted for Mars. The original version of the model is described by Anthes and Warner [1978], and the current version is described by Dudhia [1993]. The model is nonhydrostatic and uses time split-explicit integration. The model uses an Arakawa "B" grid, where temperature and pressure are calculated at grid points at the center of a box and the winds are calculated at the corners of the box. The MM5 uses three different types of map projections: Mercator, Lambert conformal, and polar stereographic. In each case, placement of grid points is constrained to form squares in the particular map projection chosen for the given simulation. The model also uses terrain-following sigma coordinates, with an upper boundary set by the user. Currently, a top at the $5 \mathrm{~Pa}$ pressure surface $(\sim 50 \mathrm{~km})$ is used. The model allows for arbitrary domain specification (using three different map projections) and for multiple domain nesting, which creates higher-resolution areas within the coarser grid. These higher-resolution domains can be nested one within each other up to a maximum of four times. Nesting can be undertaken in the model in either a "one-way" or "two-way" mode. In the one-way mode, output from a previous simulation is used to generate boundary and initial conditions for a higher-resolution nest, analogous to the way GCM boundary conditions are imposed (see below). In the two-way mode the higherresolution nests exchange information on a time step by time step basis. For all simulations discussed in this paper, and indeed most simulations in general, the two-way nesting is utilized.

[6] The initial and boundary conditions are provided by the Geophysical Fluid Dynamics Laboratory (GFDL) Mars General Circulation Model (GCM), described in section 2.2. The details of the coupling are described in section 2.3. The upper boundary condition is a constant pressure surface, with no air or aerosol exchange across the surface. This is an inherent design feature of the MM5 model and is valid in the terrestrial case since the model domain typically extends to the tropopause, where exchange is minimal. In our simulations we have taken care to extend the model top to altitudes at which the temperature profile becomes more nearly isothermal (roughly $40-50 \mathrm{~km}$ [see, e.g., Zurek et al., 1992]). Clearly, there are circumstances where having flow through the upper boundary is desirable, and we are currently working on implementing this feature.

[7] Conversion of the model to Mars involved three different types of modification. First, we made structural changes within the model related to the time integration of the various forcing functions. These included the planetary rotation and orbital revolution periods and modification of the model's definition of a "day" and a "year." We also replaced the model's orbital code which generates the daily and seasonal cycles of solar insolation. Second, various constants within the model such as the planetary radius, the Coriolis parameter, the gravitational constant, the gas constant of the atmosphere, and the solar constant were modified. Third, wholesale replacement of parameterizations for physical processes which are significantly different on Mars such as radiation, the surface and subsurface heat balance model, the $\mathrm{CO}_{2}$ cycle, the water cycle, and the dust cycle were made. In all cases the Mars-specific and scale-independent parameterizations are taken directly from the version of the Geophysical Fluid Dynamics Laboratory (GFDL) Mars General Circulation Model (GCM) described by Wilson and Hamilton [1996].

[8] The model includes the radiation scheme used in the Wilson and Hamilton [1996] version of the Geophysical Fluid Dynamics Laboratory (GFDL) Mars General Circulation Model $(\mathrm{GCM})$. This radiation scheme treats solar absorption by $\mathrm{CO}_{2}$ gas using a parameterized band model [Burk, 1976] and by atmospheric dust using a two-stream model [Briegleb, 1992]. The optical depth used in the radiation code is derived from dust tracers of two particle sizes that are advected and diffused by the model dynamics. In the infrared, radiative heating due to $\mathrm{CO}_{2}$ is treated using the band model of Hourdin [1992]. For dust the infrared scheme developed by Haberle et al. [1982] is used, and again, the optical depths derived from the model dust tracers are used. The optical properties for dust are the same as used by Wilson and Hamilton [1996]. Radiative effects due to water ice and $\mathrm{CO}_{2}$ ice are not treated. In the case of $\mathrm{CO}_{2}$, this is justified as $\mathrm{CO}_{2}$ ice will form only in the depths of polar night. Water ice may play a role under certain circum- 
stances, such as in frontal cloud systems and in the tropical cloud belt in northern summer. We anticipate incorporating water ice radiative effects in the future. It is important to note that none of the published Mars GCMs to date include radiative effects due to ice aerosols as well.

[9] The surface models used were topography derived from the Mars Orbiter Laser Altimeter (MOLA); albedo maps of the equatorial regions are from Pleskot and Miner [1981], and those of the polar regions are from Paige et al. [1994] and Paige and Keegan [1994]; and ground thermal inertia maps of the equatorial region are from Palluconi and Kieffer [1981] (as modified by Haberle and Jakosky [1991]), and those of the polar regions are from Vasavada et al. [2000]. The ground temperature calculation scheme uses a 12 layer subsurface heat diffusion model that captures the annual and seasonal temperature waves by simulating the uppermost $2 \mathrm{~m}$ of the subsurface. The subsurface layer temperatures are initialized from the GCM input and are implicitly integrated (as implemented in the GCM [Wilson and Hamilton, 1996]). It is important to note that the model currently does not include the radiative effects of slope.

[10] The model has been modified to handle the presence of interactive tracers, such as dust particles, which are used in the radiation scheme. Two dust particle sizes are currently used as described by Wilson and Hamilton [1996], although this will be expanded to a greater number in the future. The water cycle is also simulated in the model, including water vapor transport, atmospheric ice formation, transport, and precipitation, and the formation of surface ice deposits. These processes are taken from Richardson [1999] and used in place of the various hydrological cycle parameterizations included in the terrestrial version of the MM5. In the case of transport of dust, water vapor, and water ice, the tracer transport dynamics built into the MM5 were used unmodified. $\mathrm{CO}_{2}$ ice is not treated as an aerosol in the current version of the model.

[11] The MM5 boundary layer option we employ in our simulations is the Medium Range Forecast (MRF) scheme, based on the one used in the National Center for Environmental Prediction (NCEP) Medium Range Forecast (MRF) model. It is described by Hong and Pan [1996] and is based on the formulation by Troen and Mahrt [1986]. This parameterization of the boundary layer is modified only by the coupling to the calculation of surface temperatures and heat fluxes determined by the Mars subsurface model. Even in the very highest vertical resolution simulations, we do not fully resolve the spectrum of turbulent motions. As the resolution increases, an increasing fraction of the spectrum is captured explicitly by the model. However, there is still the need to represent the effects of the remaining unresolved turbulence for which the boundary layer parameterization is used.

[12] The model time step is highly variable depending on the chosen model resolution (both horizontal and vertical). Typically, it is of the order $10^{-1}$ to $10^{2} \mathrm{~s}$. Model output is also user-definable; typically, output is written once every hour for all model variables.

\subsection{GCM Description}

[13] The mesoscale model requires a description of both initial and boundary conditions. As implemented in this study, the mesoscale model is driven by boundary conditions which evolve with a 2 hour time step. These initial and boundary conditions are derived from the Geophysical Fluid Dynamics Laboratory (GFDL) Mars General Circulation Model (GCM) [Wilson and Hamilton, 1996]. Compatibility between the Mars MM5 and the GFDL Mars GCM is maximized by the use of common physical parameterizations in both models. These include the treatment of radiation, dust injection, surface and subsurface heat balance and diffusion, planetary orbit, and condensation/sublimation of $\mathrm{CO}_{2}$, including the treatment of surficial $\mathrm{CO}_{2}$ ice. These schemes have been described in section 2.1, and their description is not repeated here. Additionally, the Mars MM5 includes a full water cycle, which is again based on that in the GFDL Mars GCM [Richardson, 1999]. As water is not considered in this study, description of water processes is deferred to a later paper.

[14] The GFDL Mars GCM differs from the Mars MM5 in treatment of large-scale dynamics, sub-grid-scale diffusion, and the planetary boundary layer. The most obvious difference in the treatment of large-scale dynamics is the use of the primitive equations in the GCM, which filters out vertically propagating sound waves by employing a hydrostatic approximation for the vertical momentum equation. In addition, purely horizontally propagating sound waves (Lamb waves) are filtered out by setting vertical velocity to 0 at the surface. The GCM also treats Coriolis acceleration as a purely horizontal process (producing horizontal accelerations due to horizontal winds). These approximations are based on the small values of vertical acceleration on large scales and on the negligible heat and momentum transports due to sound waves on large scales. Another difference is the model grid structure. The GCM calculates all variables at the same horizontal grid point (this is the Arakawa "A" grid, as opposed to the "B" grid used in the MM5 [Arakawa and Lamb, 1977]) and employs a mixed sigma/pressure vertical structure, such that the vertical coordinate is terrain-following in the lower domain and is pressure in the upper portion. The GCM domain extends up to $\sim 85 \mathrm{~km}$ in order to fully capture the southern summer Hadley circulation [Wilson, 1997]. Sub-grid-scale mixing away from the surface layer is treated in the vertical as a diffusive processes acting on heat and momentum with a Richardson number-dependent coefficient. The scheme is described by Hamilton et al. [1995]. In the horizontal, mixing is dependent on the flow curvature, as described by Andrews et al. [1983]. No explicit treatment of the boundary layer is included above the surface layer beyond that which results naturally from the diffusion schemes. The surface layer is treated with a drag coefficient scheme which is based on Monin-Obuhkov theory, in which the fluxes of momentum and heat at the surface depend on the total wind speed, the Richardson number, the height of the lowest model level, and the roughness length [Wilson and Hamilton, 1996].

\subsection{Coupling of the Mars MM5 With the GCM}

[15] The Mars MM5 is a limited area model. As such, it needs boundary and initial conditions to integrate the equations of motion, energy, and mass. These are provided by the GFDL Mars GCM (as described above) through a series of "preprocessing" steps. For the simulations discussed in this 
paper, we extracted two- and three-dimensional (3-D) fields from the GCM at 2 hour intervals. These fields included the three-dimensional winds, temperature (both air and subsurface), pressure, water vapor amount, and dust amount for both particle sizes. The two-dimensional fields include surface ice (water and $\mathrm{CO}_{2}$ ) amount, surface temperature, and surface pressure. All of the above mentioned variables are used for initial conditions; only the three-dimensional fields are necessary for boundary conditions.

[16] Preprocessing consists of three steps. The first is interpolating the GCM output to constant pressure levels. It is necessary to use pressure as an intermediary vertical structure as the sigma coordinate used by the GCM and MM5 is by definition dependent on local topographic elevation. As the surface boundary for any given location in the MM5 is not constrained to be the same as that in the GCM, interpolation in sigma coordinates can be physically incorrect. After interpolation to constant pressure levels, the output is trimmed to the horizontal extent of the mesoscale model domain to be used, and the coarser GCM output is interpolated to the higher-resolution mesoscale model grid points. The vertical coordinate used in the mesoscale model is the terrain-following sigma coordinate [e.g., Jacobson, 1999]. The vertical levels to be used in a given simulation are chosen at this point. Vertical interpolation from the constant pressure levels to these sigma levels is then done. Sufficient boundary conditions must be generated for the entire mesoscale model simulation at this preprocessing stage. For the GCM comparison simulations, this was done for 10 days, while for the lander comparison simulations, the amount of time chosen was 5 days. Since the model does not start from rest (e.g., no winds and an isothermal temperature structure), there is no traditional "spin-up" time. However, experience shows that the first day of integration is affected by adjustment from the initial conditions to a balanced higher-resolution simulation. This timescale is roughly consistent with the radiative timescale of the Martian atmosphere.

[17] It should be noted that tracers such as dust and water vapor are passed into and out of the model domain via the boundary conditions. In addition, surface sources of these materials exist. The same applies to the total air mass within the model domain. The ability to transport air across the boundaries (i.e., allowing for a net divergent wind) allows the simulation of tidal propagation, and sublimation from or condensation onto the polar caps.

\section{Comparison to the Mars GCM}

[18] As a first test of the Mars MM5, a comparison to the GFDL Mars GCM on similar length scales was performed. Conversion of the MM5 to Mars involved significant modification of a number of model components. Since the majority of these components are common to the Mars MM5 and the GFDL Mars GCM, an important way to test the validity of the conversion was to compare the two models in as similar a way as possible. Such a comparison also provides a test of the validity of the Mars MM5 dynamical core (the integration of the fundamental fluid dynamical equations) for the simulation of synoptic scale dynamical processes. In both cases the validity is gauged by the degree to which the Mars MM5 can reproduce the dynamical behavior predicted by the well-tested
GFDL Mars GCM. While direct comparison to data will be discussed in later sections, it is important to note that the Mars GCM simulation used for comparison in this section compares well to Viking Infrared Thermal Mapper (IRTM) and surface pressure observations [Wilson and Hamilton, 1996; Wilson and Richardson, 2000], and thus this section can be considered a discussion of indirect comparison between the Mars MM5 and global data sets.

[19] The GCM has a horizontal resolution of $5^{\circ}$ in latitude and $6^{\circ}$ in longitude with the lowest layer being roughly $400 \mathrm{~m}$ in thickness. The GCM has 20 vertical levels between the surface and roughly $85 \mathrm{~km}$. The Mars MM5 was thus run with a horizontal resolution of $\sim 5^{\circ}$ in both latitude and longitude. The actual domain is a Mercator projection, and thus the latitudinal separation between grid points decreases somewhat with latitude. As the Mars MM5 cannot be run in a truly global mode, we attempted to make as large a domain as possible. In this case the model domain extends a full $360^{\circ}$ in longitude, although there is no connection between the easternmost and westernmost extreme grid points, that is, the model does not wrap around at the edges. These edges are fed by boundary conditions from the GCM. The latitudinal extent of the mesoscale domain ranges from $60^{\circ} \mathrm{S}$ to $60^{\circ} \mathrm{N}$. Eight vertical levels were used in the Mars MM5 simulation from the surface to roughly $50 \mathrm{~km}$, with a lowest layer thickness equivalent to that of the GCM. About 12 GCM levels fall within this vertical extent.

[20] The Mars MM5 was initialized with output from the GFDL Mars GCM. After a 10-day integration we examined the drift between the two models. There are a number of potential reasons why the two models may differ in their simulation of the circulation. These include difference in grids (Arakawa "A" grid and rectangular boxes in the GCM and Arakawa "B" grid and square boxes in the Mars MM5), differences in boundary layer schemes, subtle differences in numerical integration method, and the treatment of the atmosphere as hydrostatic in the GCM and as nonhydrostatic in the Mars MM5. However, the numerical framework should not significantly influence the simulation of the circulation of the atmosphere if it is an accurate model. Thus differences between the GCM and Mars MM5 should be small, and their comparison provides one way of testing the validity of the Mars MM5. Thankfully, the agreement between the two models is quite good and generally traceable to subtle differences in the strength of the Hadley cell flow between the two models.

[21] We conducted two comparisons between the GCM and the Mars MM5 at two dates: $L_{s}=180$ (equinoctial period) and $L_{s}=270$ (solstitial period). Figures 1 and 3 show output from the GCM and Mars MM5 as well as their differences for the $L_{s}=180$ and $L_{s}=270$ comparisons, respectively. Temperature and winds are from the surface model layer, $\sim 400 \mathrm{~m}$ in thickness. Figures 2 and 4 show latitude- and height-dependent output for the same simulations as zonal averages. Temperature, zonal wind, meridional wind, and vertical wind are shown.

\subsection{Equinox}

[22] The near-surface air temperatures displayed in Figures $1 \mathrm{a}$ and $1 \mathrm{~b}$ generally agree to within $5 \mathrm{~K}$ between the two models. This level of agreement is gratifying given the over $60 \mathrm{~K}$ amplitude of the diurnal cycle and nearly $100 \mathrm{~K}$ pole-to- 
A

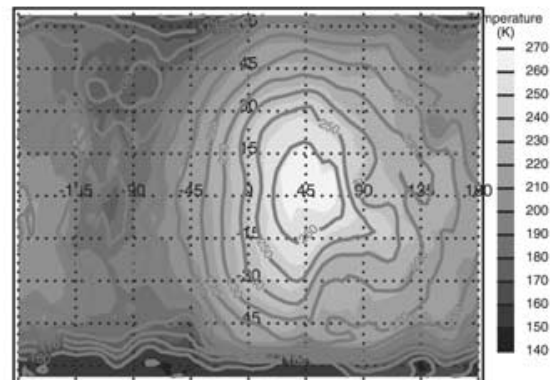

C

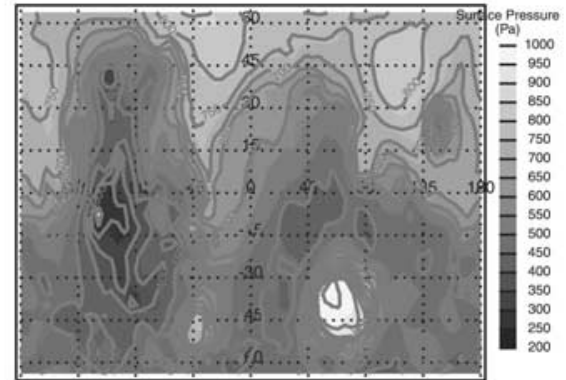

$\mathrm{E}$

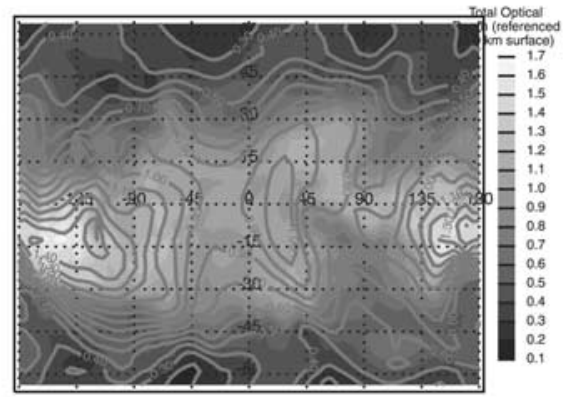

G

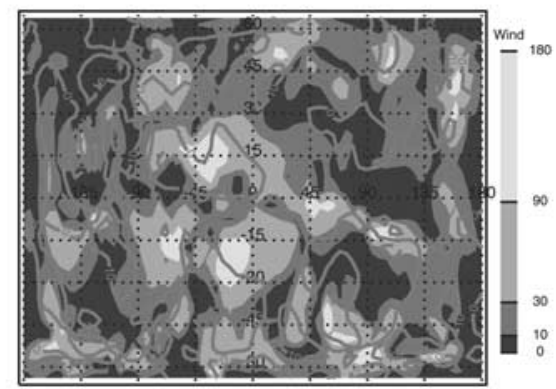

B

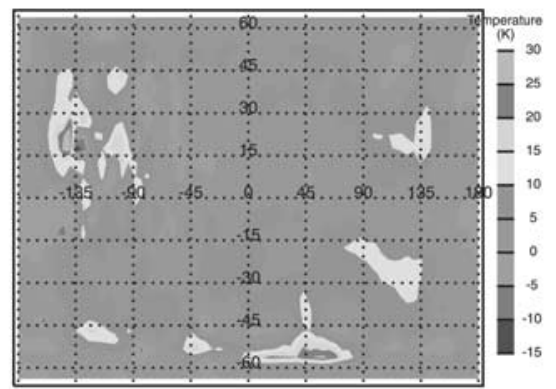

D

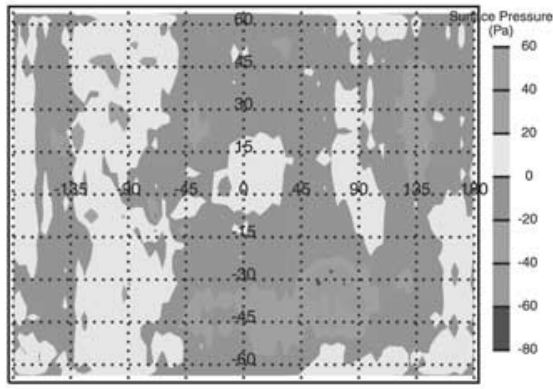

$\mathrm{F}$

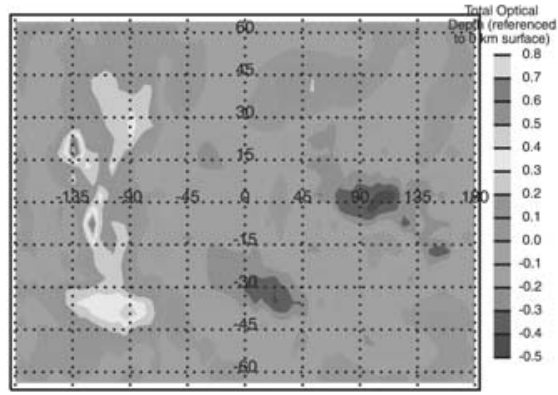

$\mathrm{H}$

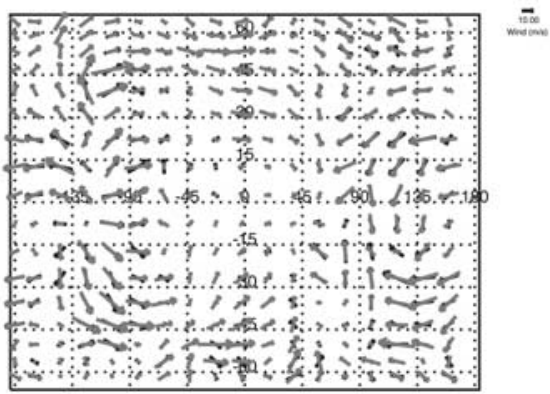

Figure 1. Map projections of model output for various variables at $L_{s}=180$. The local time is noon at $0^{\circ}$ longitude. For the upper six plots, the left-hand column is GCM and Mars MM5 output plotted on top of each other, with the GCM output in the background as a gray shading and the Mars MM5 overplotted as contours. The right-hand column is the difference of the output, Mars MM5 output minus GCM output. ( $\mathrm{a}$ and b) Temperature. (c and d) Surface pressure. (e and f) Total visible optical depth (referenced to the $0 \mathrm{~km}$ surface). ( $\mathrm{g}$ ) The absolute difference in wind direction, in degrees, is plotted in the background as a gray shading, with ranges labeled by the scale bar at the right. The red contours represent the difference in wind speed between the two models (Mars MM5 minus GCM). (h) Wind vectors for the GCM (plotted in black) and the Mars MM5 (plotted in red). Scale bar for wind speed is at the upper right. See color version of this figure at back of this issue.

equator temperature contrast. The largest differences are over the Tharsis region and near the cap edge. The latter are mostly due to slight differences in representation of the location of the cap edge, related to the difference in the placement of grid points between the two models. The circulation around
Tharsis is inherently difficult to simulate on synoptic scales (hundreds of kilometers) due to the large variability of and large gradients in topography on these scales. Thus differences in simulating the circulation over this region between the two models is not particularly surprising. 

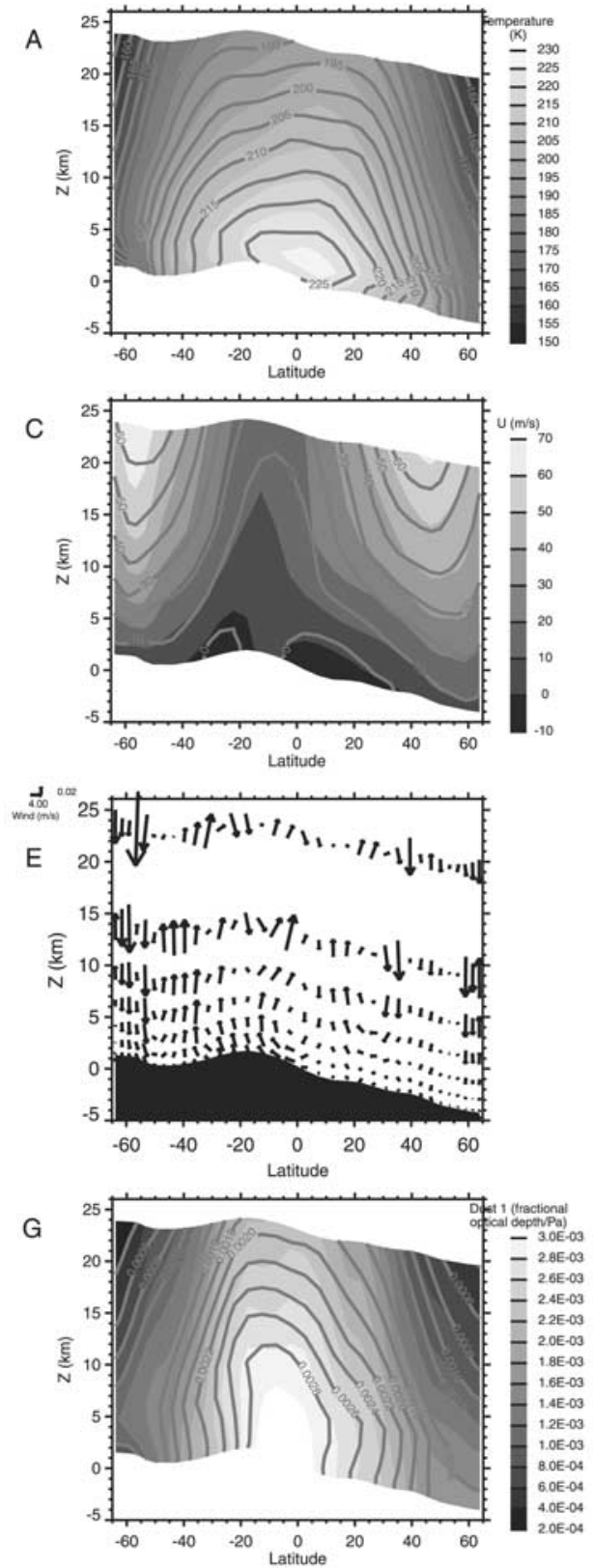
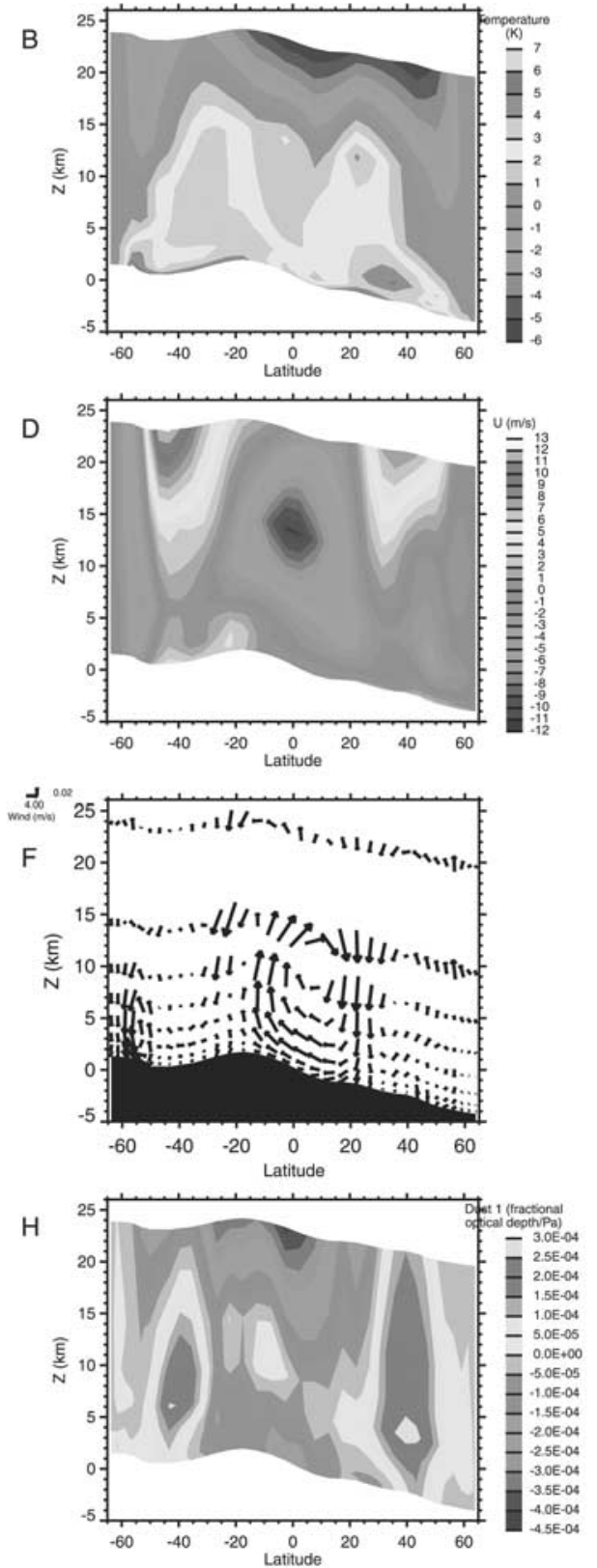

Figure 2. Zonal averages of model output, plotted as latitude versus height, for $L_{s}=180$. Except for the wind vector figures, the left-hand column represents GCM data in the background as a gray shading with Mars MM5 data overplotted in red contours, and the right-hand column is the difference between the two models, Mars MM5 minus GCM. (a and b) Temperature. (c and d) Zonal wind. (e and f) Mean meridional circulation. The vertical velocities have been exaggerated by a factor of 200 , and appropriate vector scale bars are at the upper left of the figures. (e) GCM. (f) Mars MM5. (g and h) Dust amount. The units of dust used here are fractional optical depth over the grid box horizontal area per unit thickness of the grid box in pressure. See color version of this figure at back of this issue.

[23] Differences in surface pressure are rather small, within $\pm 20 \mathrm{~Pa}$ over most of the globe. The largest differences occur over regions of large topography (i.e., Hellas basin and Tharsis), which, again, is likely related to the difference in grid point positioning along topographic gradients. The modeled surface pressure outputs are shown in Figures 1c and 1d.

[24] The distribution of dust is the most difficult field to accurately simulate. This is because the distribution of dust is both sensitively dependent on the circulation and modifies the distribution of radiative heating, which in turn modifies the circulation. Thus this field provides a very sensitive test of the coupled radiative-dynamical behavior of the two models. Figures 1e and 1f show the modeled visible optical depth normalized to the $0 \mathrm{~km}$ reference surface. As discussed in section 2, dust is passed into the mesoscale model domain by the boundary conditions and is also injected from the surface within the mesoscale model domain using a surface/air 
temperature contrast criterion. Agreement is to within \pm 0.2 for roughly three quarters of the modeled domain, and the biggest differences occur in the regions where the gradient in optical depth is largest. Generally, the GCM has more dust, and it is more equatorially confined. The Mars MM5 has a slightly smoother distribution with more dust toward the poles. As we shall discuss when we examine the zonal average fields, the Mars MM5 may be exporting more dust from the tropics to the midlatitudes. The most significant discrepancies are along the western edge of Tharsis, where the Mars MM5 has temperatures and surface pressures larger than the GCM. The larger dust amounts in this region result from a more active boundary layer driven by the higher temperatures and from the ability of the atmosphere to hold more dust due to the higher pressure.

[25] The synoptic scale flow patterns are similar in the two models as shown in Figures $1 \mathrm{~g}$ and $1 \mathrm{~h}$. The surface wind patterns in both models (Figure $1 \mathrm{~h}$ ) are dominated by the tidal flow as modified by topography, with convergence lagging the daily temperature maxima and divergence roughly $180^{\circ}$ out of phase. The largest differences occur again at the regions of large topography, Hellas basin and Tharsis. On the whole, directional agreement between the mesoscale model and the GCM is within $30^{\circ}$, and agreement in speed is within $10 \mathrm{~m} / \mathrm{s}$. Areas where discrepancies are larger are restricted to regions of lowest wind speed. Consequently, relatively small differences in the magnitude of the wind component vectors can translate into large angular differences.

[26] Figures $2 \mathrm{a}$ and $2 \mathrm{~b}$ show the zonal average temperatures for the two models. As with the near-surface air temperature, the general agreement is quite good. The primary difference occurs at the upper levels over the equator. Here the Mars MM5 is as much as $6 \mathrm{~K}$ cooler than the GCM. Examining Figures $2 \mathrm{~g}$ and $2 \mathrm{~h}$, we can see that the amount of dust at the upper levels of the model domain is less in the Mars MM5 as compared to the GCM. Thus the primary explanation for the large temperature differences at high levels is differences in solar heating due to absorption by dust.

[27] Referring back to Figure 2b, we also find a temperature difference of $<5 \mathrm{~K}$ at midlatitudes from the surface to roughly $15 \mathrm{~km}$. The existence of these temperature deviations results from two factors: (1) The presence (see Figure 2f) of a more confined and stronger Hadley circulation (as compared to the GCM) results in adiabatic descent and warming at the midlatitudes. (2) As mentioned in the optical depth discussion, the existence of more dust in the Mars MM5 at midlatitudes and high latitudes results in direct radiative heating.

[28] The zonal winds are shown in Figures $2 \mathrm{c}$ and $2 \mathrm{~d}$. Both models maintain a strong polar jet, and only small differences in the width of the jets yield differences $(\leq 15 \%)$ in zonal wind speed. The Mars MM5 has broader and weaker jets in both hemispheres. The increased width of the jets suggests a somewhat stronger meridional momentum mixing process in the Mars MM5. Indeed, stronger mixing, especially across the polar fronts, is also evident in the comparison of dust distribution (Figures $2 \mathrm{~g}$ and $2 \mathrm{~h}$ ), with the dust distributed more poleward in the Mars MM5. This increased mixing in the upper portion of the mesocale model appears to be associated with the proximity of the rigid (though free-slip) lid. Note that the Mars MM5 model top is significantly lower than that of the GCM (50 km versus $85 \mathrm{~km})$. When the simulation is repeated with a domain of higher vertical extent, the widths of the Mars MM5 jets are observed to decrease (not shown). In fact, in this case the jets become slightly more confined than in the GCM.

[29] Figures $2 \mathrm{c}$ and $2 \mathrm{~d}$ also show a zonal wind deficit in the Mars MM5 at midlevels $(\sim 15 \mathrm{~km})$ over the equator. The occurrence of this deceleration of the westerlies (by up to 10 $\mathrm{m} / \mathrm{s}$ ) is consistent with the vertical transport and deposition of momentum by a shallower upwelling branch of the Hadley cell.

\subsection{Solstice}

[30] Most of the comments made about the surface and column integrated characteristics of the fields at equinox apply equally well at the solstice (Figure 3), for example, the surface temperature and pressure differences between the models. A slight difference from equinox is in Arabia Terra, where the Mars MM5 shows more dust at the northern edge of this area and less dust in the interior. The excess dust on the northern edge of Arabia Terra occurs at the location of greatest gradient in optical depth at the polar front, which probably represents a slight difference in the latitudinal location of the polar vortex wall and hence a slight poleward expansion of the dusty extratropical air mass. The difference in dust amount in the interior region of Arabia Terra appears to be due to the inability of the Hadley cell circulation to deliver dust to this northern subtropical region, as discussed below.

[31] The solstice mean meridional circulation (Figure 4) is dominated by a much stronger and latitudinally more extensive Hadley cell than during equinox, but the circulation is still more confined in the Mars MM5 as compared to the GCM, due to the presence of a rigid lid. Examples of this can be seen in the mean meridional circulation (Figures $4 \mathrm{e}$ and 4f), where the upwelling at about $20^{\circ} \mathrm{S}$ is weaker in the Mars MM5, especially at the top layer. This is also reflected in the zonal average temperature pattern, where there are higher temperatures in the upwelling branch of the Mars MM5 due to decreased adiabatic cooling and cooler temperatures at upper levels in the descending branch. However, the signal is most clear in the meridional transport of dust, where dust accumulates in the upwelling branch and is depleted relative to the GCM in the downwelling branch. Note the small excess at high north latitudes and low levels due to a slight poleward flow at low levels, which accumulates the dust that does make it down the downwelling branch.

[32] As seen in Figures $4 c$ and $4 d$, the polar jet is wider in the Mars MM5 as compared to the GCM for the same reasons discussed in the equinox case. The westerly excess at midlevels over the equator as compared to the GCM now results from weaker upward momentum transport in contrast to the equinoctial case.

[33] In summary, after 10 days of integration, the level of agreement is pleasing. The minor differences that do exist are easily explained by the intrinsic design of the mesoscale model as a limited area model (in the horizontal and vertical), leading to a more confined Hadley circulation. This is not a major difficulty, so long as these factors are borne in mind when designing numerical experiments with the model. Most of the studies to be undertaken with the model will relate to near-surface flow phenomena. Within 
A

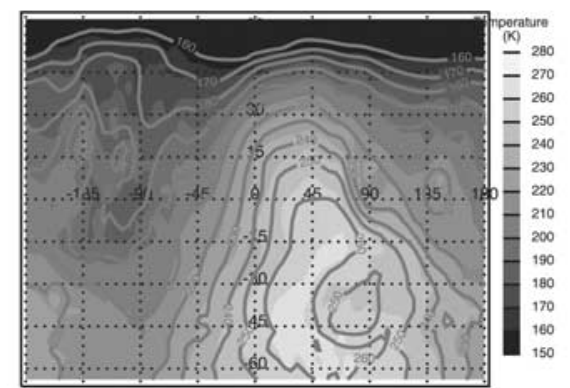

C

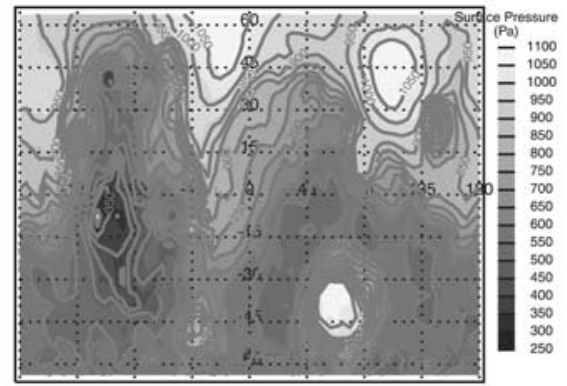

$\mathrm{E}$

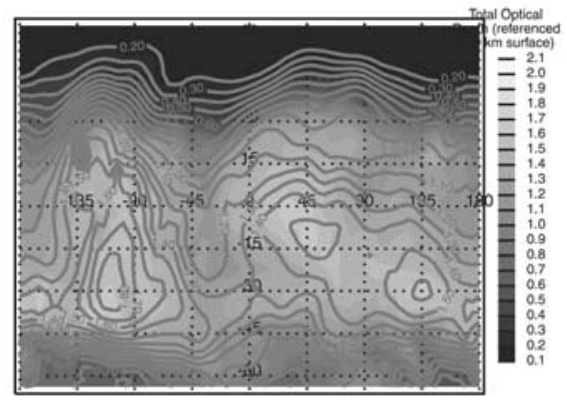

G

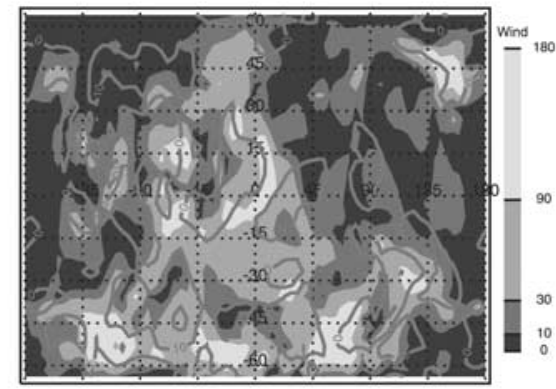

B

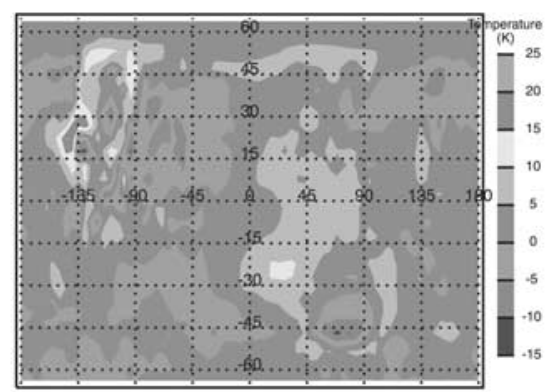

$\mathrm{D}$

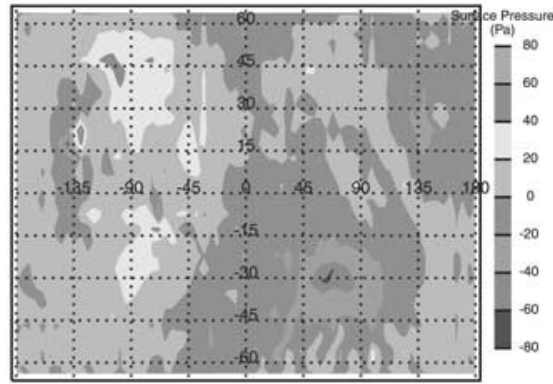

$\mathrm{F}$

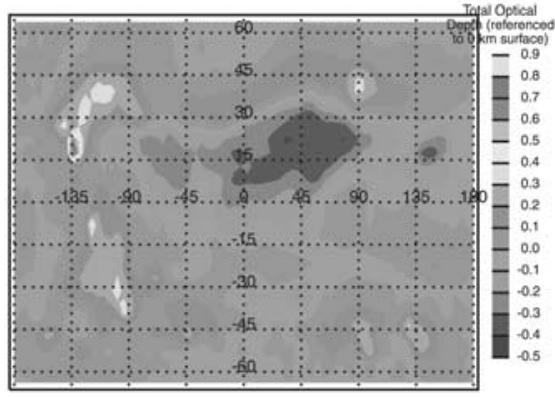

$\mathrm{H}$

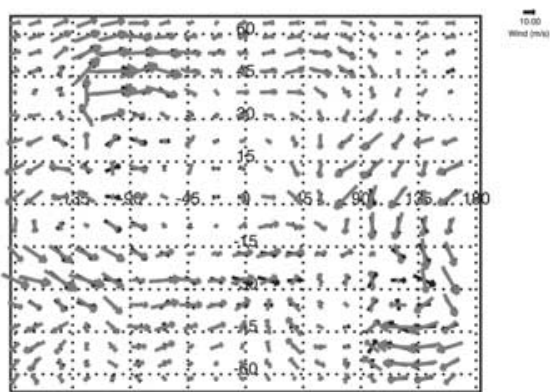

Figure 3. Same as Figure 1, except for $L_{s}=270$. See color version of this figure at back of this issue.

this region of the atmosphere the circulation is dominated by the surface and by tidal flow, and thus the details of the upper level Hadley flow are less important [Wilson and Hamilton, 1996; Joshi et al., 1997]. However, in cases where deeper atmospheric circulation phenomena are to be studied (e.g., modeling the polar vortex), attention must be paid to creating a model domain with sufficient depth.

\section{Model Validation Against Meteorological Stations}

[34] As a further test of the validity of the model, simulations were performed to compare the Mars MM5 model output to meteorological observations from the near surface of Mars. These data are provided by the meteorological instruments on the three successful landers on Mars: Mars Pathfinder, Viking Lander 1, and Viking Lander 2. Data used in these comparisons were obtained from the Planetary Data System (PDS) lander data volumes ("VL_1001" and "MPAM_0001"). These comparisons take advantage of the particular strengths of the mesoscale model, allowing for simulations using high vertical and horizontal resolution.

[35] Simulations were performed at one specific time of year at each landing site, except for Viking Lander 2, where two times of year were simulated. The Mars Pathfinder and Viking Lander 1 simulations were performed during northern summer $\left(L_{s}=147\right.$ and $L_{s}=111$, respectively), while 

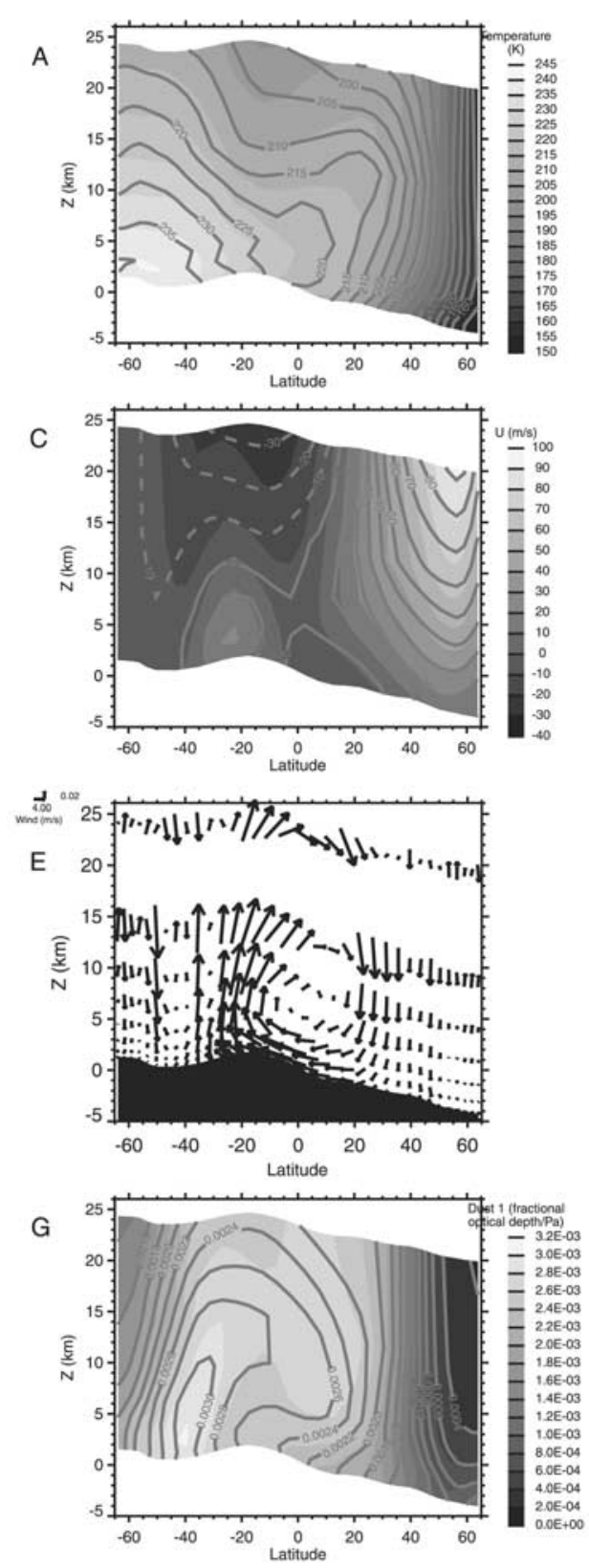
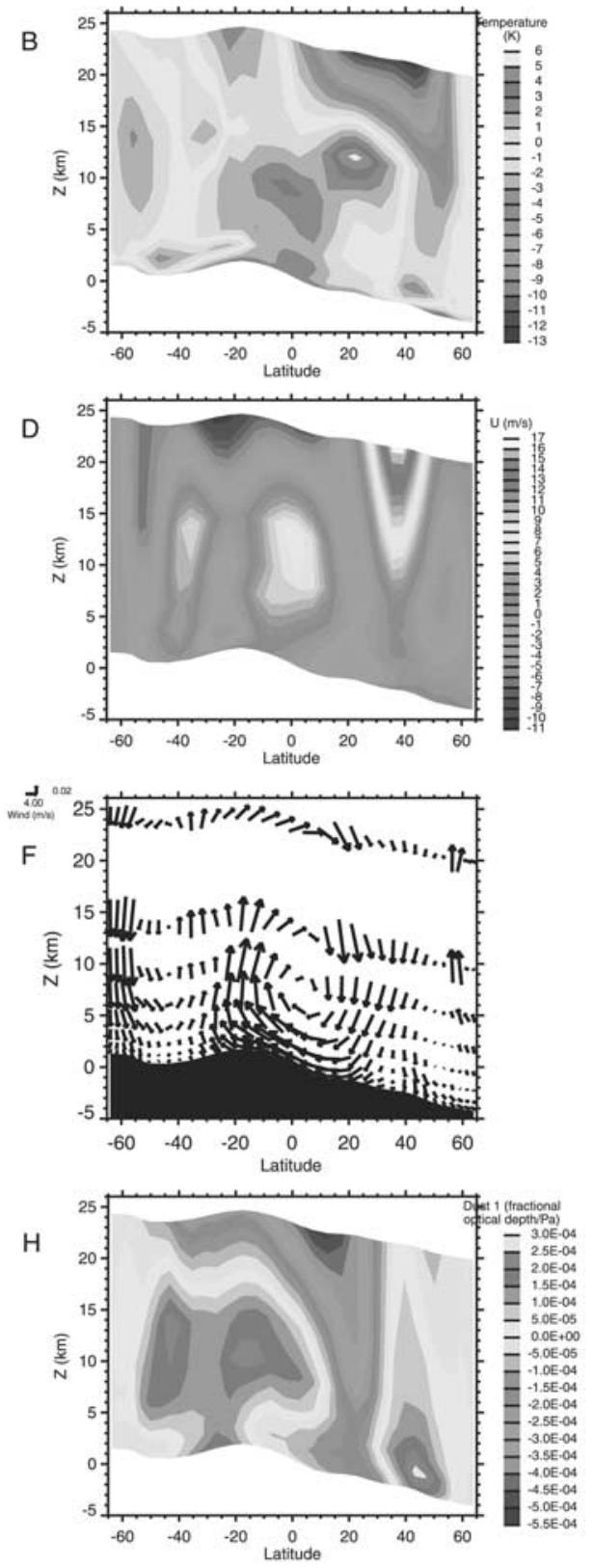

Figure 4. Same as Figure 2, except for $L_{s}=270$. See color version of this figure at back of this issue.

Viking Lander 2 simulations were carried out during both the northern summer $\left(L_{s}=130\right)$ and, for a more stringent test of the model, the northern winter period $\left(L_{s}=334\right)$. All of the simulations were designed with the same grid point structure, a 31 by 31 grid with 18 vertical levels (extending up to $50 \mathrm{~km}$ in height). The horizontal resolution was $1 / 16$ of a degree $(\sim 4 \mathrm{~km})$ in the horizontal, resulting in a square domain of length $120 \mathrm{~km}$ to a side (roughly $2^{\circ}$ ). The lowest vertical layer had a thickness of $\sim 4 \mathrm{~m}$, allowing for direct comparison to height of the meteorological instruments without having to scale for height. As noted in section 2, in contrast to the GCM, the mesoscale model is not "spunup" from rest. Consequently, the adjustment period is the roughly 1 day that is required for the slight relaxation from the initial conditions that were generated from the lowresolution GCM output. Integrations were performed for
5 days, and 2-day averages of the mesoscale and GCM model output were compared to 2-day averages of the lander observations. The averaging was undertaken to reduce the effect of day-to-day variability associated with "weather." Such weather was particularly severe in both the model output and the data at the Viking Lander 2 site during winter. For each of the landing sites one or more further simulations were performed varying a parameter to determine that parameter's effect on the simulation. The results of these tests, as well as the standard cases, will be described below.

[36] For each landing site a subset of the meteorological variables pressure, temperature, and wind velocity (speed and direction) will be compared to model output. Different landing sites, during different periods, have different availability of these variables. In all cases the output from the 

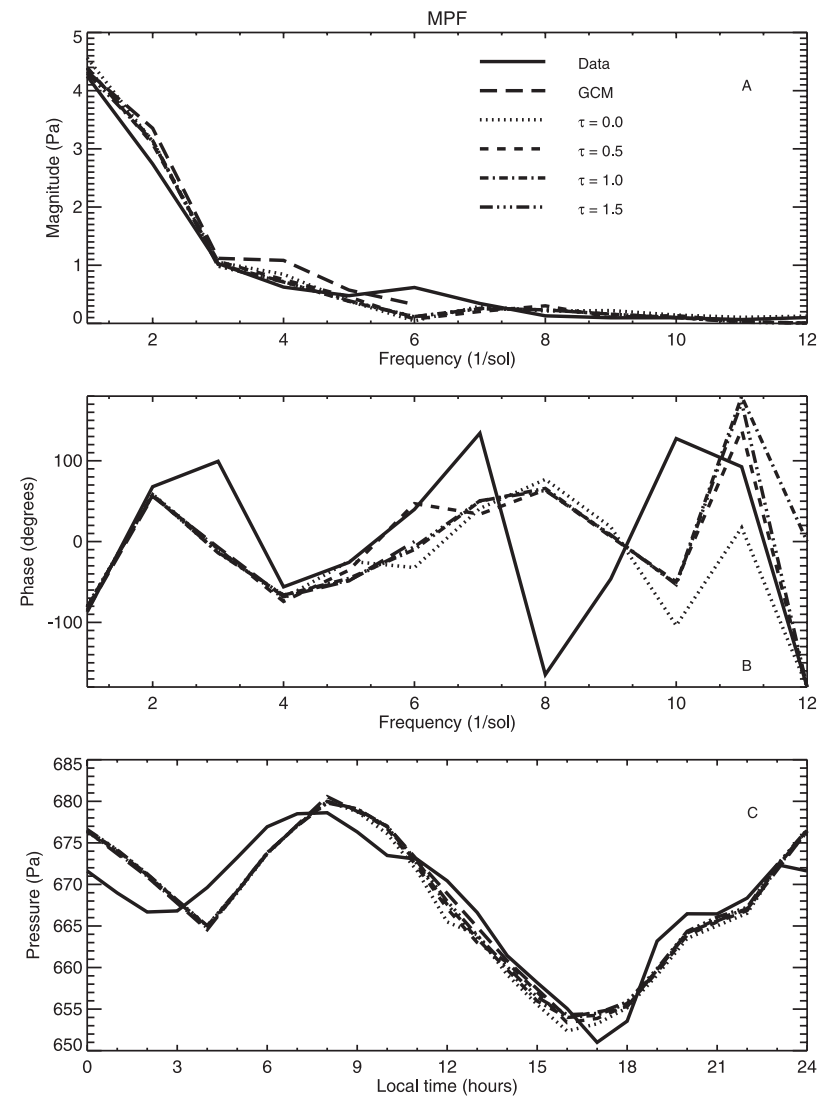

Figure 5. Comparison of pressure at the Mars Pathfinder site. (a) Amplitude of the diurnal, semidiurnal, and higherorder terms of the pressure as a function of frequency in 1/ sol, where 1 sol is one Martian day. Amplitudes were obtained by taking the Fourier transform of the output from the model simulations (both GCM and Mars MM5) and of the lander measurements. Lander measurements come from sol 9 of the Pathfinder mission, approximately $L_{s}=147$. (b) Same as Figure 5a except the phase is plotted. (c) Plot of the diurnal cycle of pressure of the model simulations and the data as a function of local time in Martian hours, where 1 Martian hour is $1 / 24$ of a Martian day.

Mars MM5 simulations is the data from the lowest layer, $\sim 2$ $\mathrm{m}$ from the surface.

\subsection{Mars Pathfinder Site}

[37] Choice of the period of simulation of the Pathfinder site was heavily constrained by the short length of the mission. A period early on during the mission (the second week) was chosen since a full 24 hours of data (pressure, temperature, and wind direction) were collected. A deficiency of the data set, in comparison to the Viking Lander data sets, is the lack of retrieved of wind speeds. However, in terms of temporal resolution and precision, the quality of the other Pathfinder data relative to the Viking Lander data is higher.

[38] In varying the amount of dust in the lander simulations, we use uniform dust amounts, and not the interactive dust used in the GCM comparisons and described above, since the domain is so small that the total amount of dust is essentially uniform. We do not rerun the GCM with modified dust amounts since we are interested in examining the impact of opacity on local dynamical phenomena, holding the global-scale circulation (as determined by the Mars GCM) constant. Note that this provides an interesting test as to whether the Mars MM5 can evolve its own circulation independent of the boundary condition forcing; if not, one would generate identical Mars MM5 simulations regardless of the locally imposed opacity. A notable result is that in all cases, the optical depth at each location determined from the use of interactive dust is the most similar to the "best" uniform optical depth chosen for that location and time.

[39] Figure 5 shows the data and modeled surface pressures for a 24 hour time series. The fit is exceptionally good, as gauged by the magnitude and phase of the diurnal and semidiurnal tidal components. Errors, especially in the phase, appear at higher frequencies, where the amplitude is substantially lower. Also shown in Figure 5 is the pressure as simulated by the GCM. It is important to note that the Mars MM5 does not significantly modify the tide as driven by the GCM. Given that the tides are a global wave system, it is not surprising that simulation of a very small domain at high-resolution does not significantly alter the surface pressure response. It is, however, encouraging that the Mars MM5 is so readily able to propagate the GCM global tidal system through the model domain.

[40] Air temperature is the variable in the Mars MM5 most sensitive to optical depth amount. Shown in Figure 6 is the data and model output for a variety of optical depth cases. Using the spacecraft-derived thermal inertia and albedo values, the model is able to generate a diurnal cycle of temperature to within $5 \mathrm{~K}$ for the best case ( $\tau$ is between 0.5 and 1.0, as compared to the measured value of about 0.5 [Smith and Lemmon, 1999]). The total range of observed temperatures is about $60 \mathrm{~K}$. We consistently underpredict nighttime temperatures by about $5 \mathrm{~K}$. This may be due to errors in the thermal inertia (but see below) or in the parameterization of the subsurface heat diffusion. An inability to capture the nighttime lowest temperatures and the

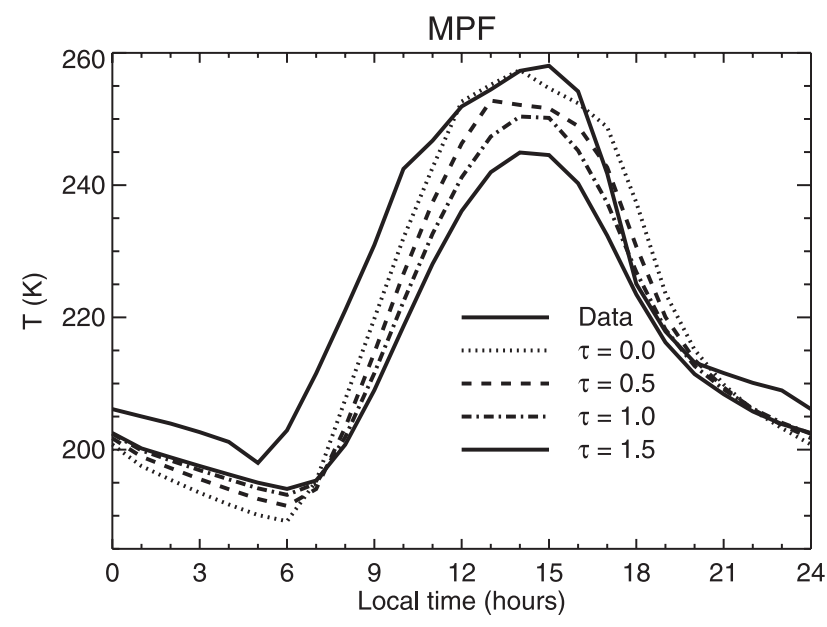

Figure 6. Diurnal temperature cycle comparison at the Mars Pathfinder site. Temperature of the air at $\sim 2 \mathrm{~m}$ from the surface is plotted versus local time in Martian hours. Data is from sol 9 of the Pathfinder mission, approximately $L_{s}=147$. 


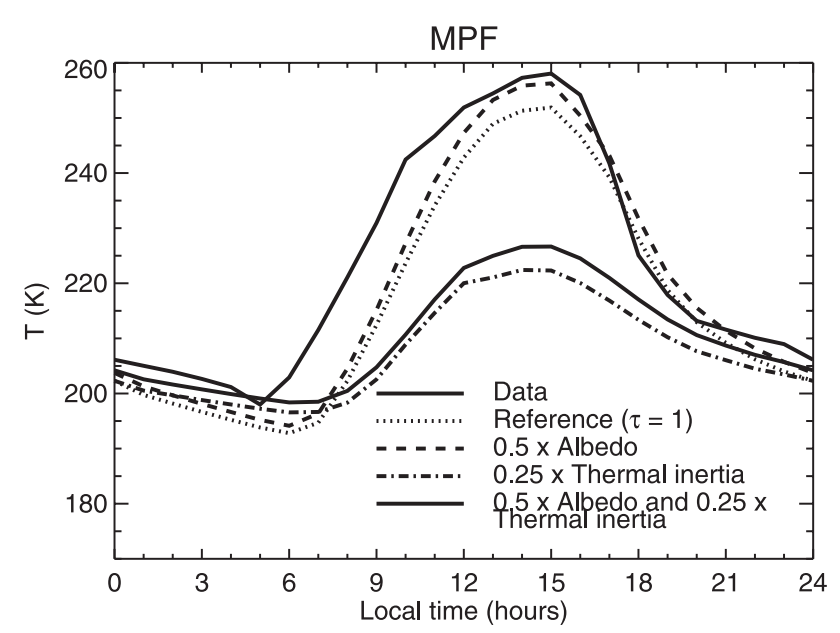

Figure 7. Diurnal temperature cycle comparison at the Mars Pathfinder site. Data are compared to the reference case using an optical depth of 1.0. The reference case is then modified in a 3 point by 3 point box in the domain around the landing site. First, the albedo is reduced by half, then the thermal inertia is reduced to a quarter of its original value, and then the third case is a combination of the first two.

timing of postdawn increase in temperatures has been noted before in a 1-D planetary boundary layer model by Wilson and Joshi [1999]. The daytime temperatures can be fit by varying the optical depth, but we attain the observed temperature maximum only with the absence of dust. We ascribe the best fit to the $\tau=0.5$ to 1.0 cases because of the fit to the total range of temperature with the view that the model cycle is simply shifted colder by $5 \mathrm{~K}$.

[41] In order to examine the effects of albedo and thermal inertia on the diurnal temperature cycle, we ran three more simulations by modifying the best fit case. The values of thermal inertia and albedo in a $10 \mathrm{~km}$ by $10 \mathrm{~km}$ box ( 9 out of the 961 total grid points) centered on the landing site were varied. In one case the albedo was reduced to half its value, in the second thermal inertia was decreased to one fourth its original value, and in the third both changes were made. These changes are intentionally large to highlight the impact of changing these parameters. The results are shown in Figure 7. Unsurprisingly, changing the albedo has very little effect on nighttime temperatures. The effect is primarily to change daytime maximum temperatures. Changing thermal inertia does in fact change nighttime temperatures; that is, a decrease in thermal inertia produces a temperature increase. And, indeed, the nighttime minimum temperature from the data is matched. However, daytime temperatures are drastically reduced. Decreasing the albedo does not make up for this drop in daytime peak temperatures. Probably equally importantly, the increase in temperatures after dawn is delayed by over an hour. This only exacerbates the preexisting mismatch in our best case, where the postdawn daytime increase in temperature is already late by about 1 hour. These results tend to rule out the effect of thermal inertia and albedo in our misfit to measured data. It thus appears more likely either that a slope effect exists or that there are slight errors in the parameterization of either subsurface or boundary layer heat diffusion. A sloping surface changes the amount of radiation absorbed at a given local time, and thus an eastward sloping surface at the Pathfinder site would tend to have an earlier rise in surface temperatures and hence near-surface air temperature. The Pathfinder site does indeed have an eastward slope [Kirk et al., 1999].

[42] Observed and simulated wind direction data are shown in Figure 8. A single day's worth of lander observations are shown but agree with longer baseline averages shown by Schofield et al. [1997]. Data for the various opacity cases and the GCM are also shown. Fits in all cases are quite good. Note that there is very little variation either among the different opacity cases or between the GCM and the Mars MM5. This suggests that the global tidal patterns and/or wind patterns generated by slopes resolvable by the GCM are more important in determining the wind directions than local slope effects resolvable only by the mesoscale model. We will return to this subject when considering the other lander sites.

\subsection{Viking Lander 1 Site}

[43] The Viking Lander 1 meteorological data also extends only over a brief period. Thus our choice of season was limited. The Pathfinder data were limited to a late summer period, and so with the Viking Lander 1 data we decided to examine a period as close to the summer solstice as possible. This turns out to be equivalent to looking at data as early in the Viking Lander 1 mission as possible. The chosen season was $L_{s}=111$.

[44] The pressure data along with Mars MM5 output at various optical depths and the GCM output are shown in Figure 9. As with the Pathfinder site, the Mars MM5 pressure output follows the GCM output very closely. In this case, however, it would appear that the diurnal and semidiurnal amplitudes are overpredicted. A significant difficulty in making this determination is that the Viking Lander 1 pressure data at this season are poorly resolved with some data gaps. Thus we have concerns about the pressure data that limit our ability to determine how well we match the data. In

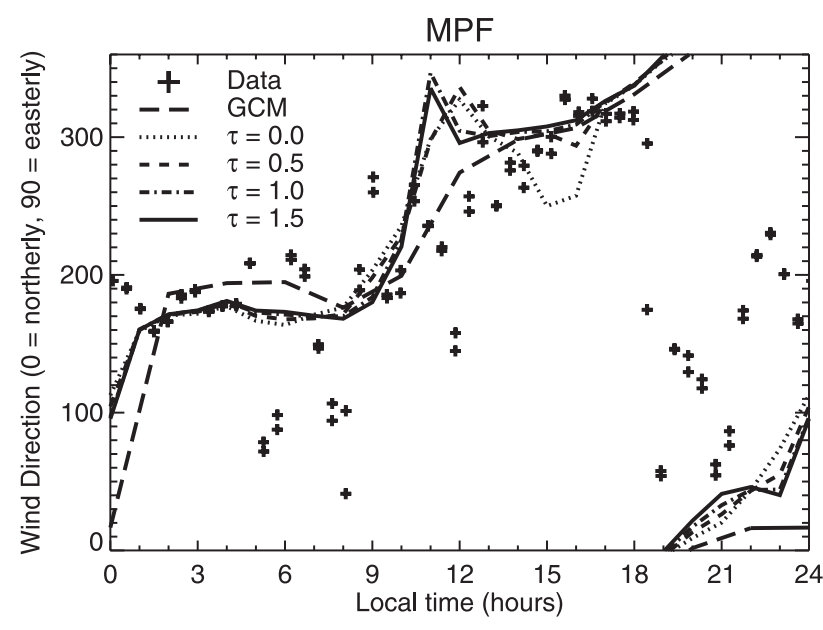

Figure 8. Wind directions at the Mars Pathfinder site. Data measured by the lander on sol 9 are plotted as crosses, and the output from the GCM and the various Mars MM5 simulations are plotted as lines. Direction is defined as 0 for a northerly (toward the south) wind, 90 for easterly, 180 for westerly, and 270 for southerly winds. Model output winds are for a height $\sim 2 \mathrm{~m}$ from the surface. 

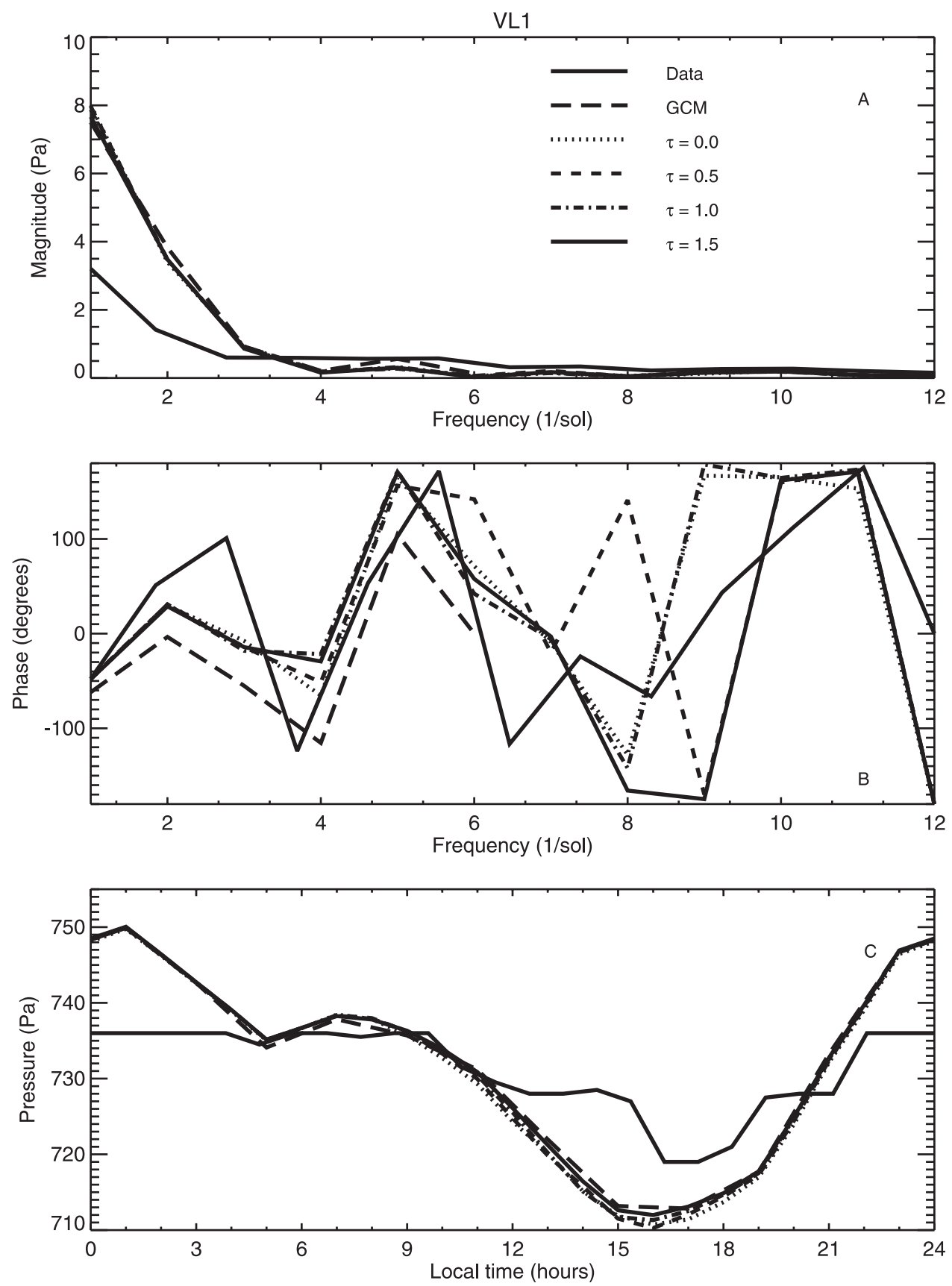

Figure 9. Same as Figure 5, except for the Viking Lander 1 site. Time of year is $L_{s}=111$.

choosing this period we had to make trade-offs among the quality and availability of the various Viking Lander 1 measurements. Good quality in one variable tended not to be correlated with good quality in the others. The determining factor in choosing this period was the availability of good wind measurements, not pressure.

[45] Near-surface air temperatures are plotted in Figure 10. The observations are shown along with the Mars MM5 output for various optical depths. The nighttime temperatures and the timing of increase in temperature (postdawn) are captured in contrast to the Pathfinder simulations. However, we do not capture the late morning flattening of the temperature increase. This may be due to underprediction of vertical heat fluxes. This effect is less obvious, but also apparent, in the Mars Pathfinder air temperature comparison (Figure 6). The various optical depth cases serve to generate a spread in daytime temperatures, and the best fit appears to be about $\tau=0.5$. This is equivalent to saying that the model is better able to fit the data with "non-dust storm" opacities but that some opacity is necessary. The step size in opacity is too large to allow more detailed discussion and tuning, which anyway is not the purpose of the comparison.

[46] Wind directions for the Viking Lander 1 site are shown in Figure 11, including the lander data, the GCM, and the Mars MM5 for the best fit dust case. All models agree pretty well with the data between 1900 and 0700 LT. In the daytime period the GCM exhibits a strong rotation between 1100 and 1300 LT which is at variance with the 


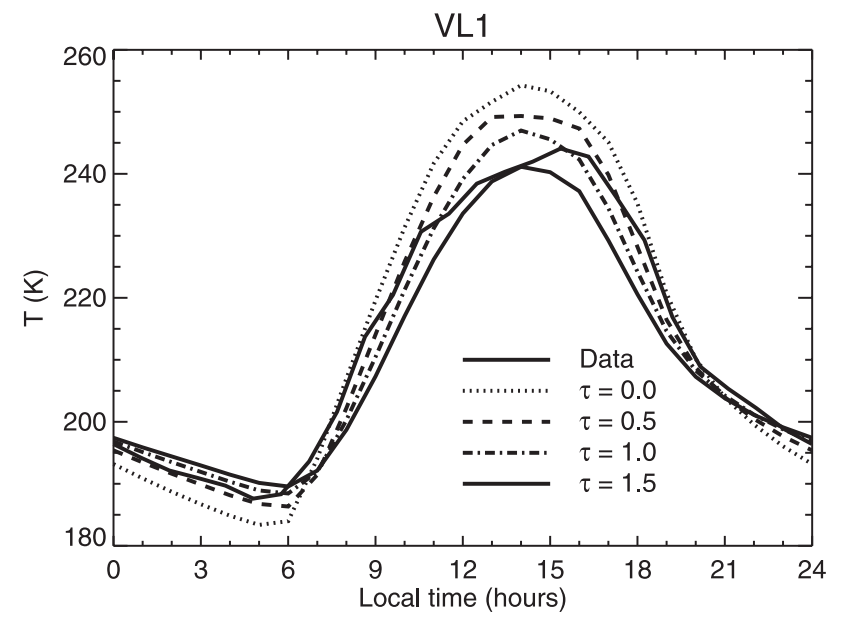

Figure 10. Same as Figure 6, except for the Viking Lander 1 site.

observations. The Mars MM5 is also at variance with observations although the pattern is less straightforward. The other dust scenarios are roughly equivalent to the best case in terms of being good fits at night and having a similar amount of variability and lack of fit, although with a different trend, during the day.

[47] The hodograph for the lander data and the best fit dust case are shown in Figure 12. The magnitudes of the wind are quite low compared to the lander data even at times when the model was correctly predicting direction. The problem of underprediction of wind speeds is common to all lander simulations that we have undertaken (see also section 4.3). To address this, we examined the vertical structure of the wind as a function of local time, as shown in Figure 13, which shows that the wind increases away from the surface as would be expected on the basis of boundary layer theory. Consequently, we experimented with increasing the vertical diffusivity in order to couple the lower level of the model more strongly to these upper

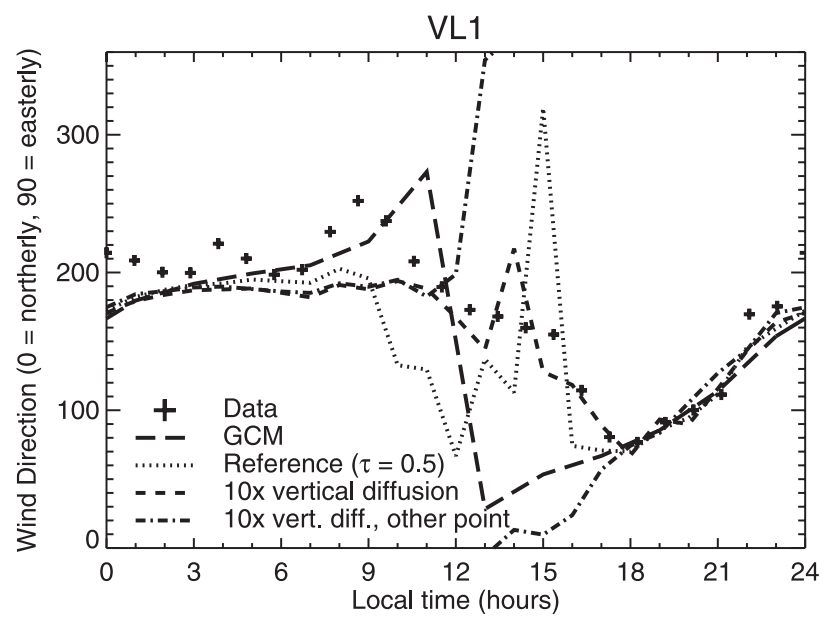

Figure 11. Same as Figure 8, except for the Viking Lander 1 site. The reference case is for an optical depth of 0.5 , and the location of the other sample point is about $27 \mathrm{~km}$ to the northeast of the reported landing site.

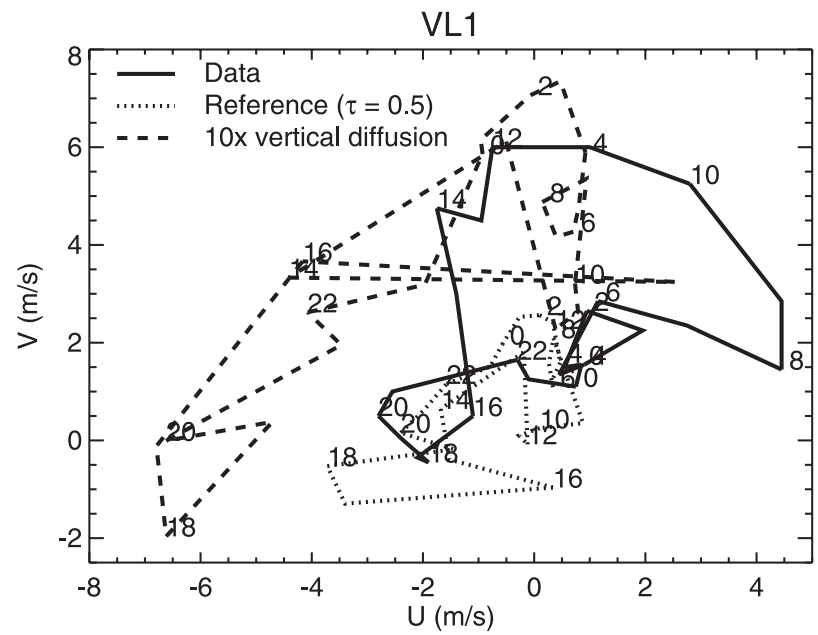

Figure 12. Hodograph of the wind velocity vectors at the Viking Lander 1 site. The numbers next to the lines indicate the local hour to which the wind vector is appropriate.

level winds. It should be noted that we compared the vertical diffusivities generated by the standard case to those reported by Savijärvi and Siili [1993] in order to confirm that there was no error in our boundary layer calculation. Indeed, we found that our model calculated vertical diffusivities very similar to those reported by Savijärvi and Siili [1993]. We proceeded to increase the vertical diffusivity by a factor of 10 and examined the impact on the model. The structure of the boundary layer with its higher diffusivity is shown in Figure 14. The wind maxima at 0300 LT that occurred below $1 \mathrm{~km}$ in the reference simulation (optical depth $=0.5$, without enhanced vertical diffusivity) has been moved up to about $1.5 \mathrm{~km}$. The location of the top of the boundary layer was not significantly affected by the increase in vertical diffusivity. This can be gauged either by examining the wind speed or temperature contours above about $3 \mathrm{~km}$ or by examining the model-predicted height of the boundary layer, which is shown as the shaded region in the figures.

[48] The impact on the near-surface wind of increased vertical diffusivity is also shown in Figures 11 and 12. Note that the simulation-to-simulation differences in wind direction variability are not associated with the choice of averaging period. The same pattern of local time wind direction variability is repeated in each simulation regardless of the length of averaging period. The differences truly represent changes in the wind direction behavior. The increased vertical diffusion significantly improves the wind directions as well as increasing the speeds to near the observed values. Unfortunately, there remains a phase shift in the relationship between the wind directions and speeds; that is, peak winds occur at different times in the model and in the data.

[49] In examining the wind directions at the Viking Lander 1 landing site, Haberle et al. [1993] were able to fit the wind velocities with a one-dimensional slope-wind model, but only by using a slope of different direction to that inferred from the pre-MOLA topography data. The slope direction and magnitude used by Haberle et al. [1993] differ from those derived from the MOLA 1/16 degree 


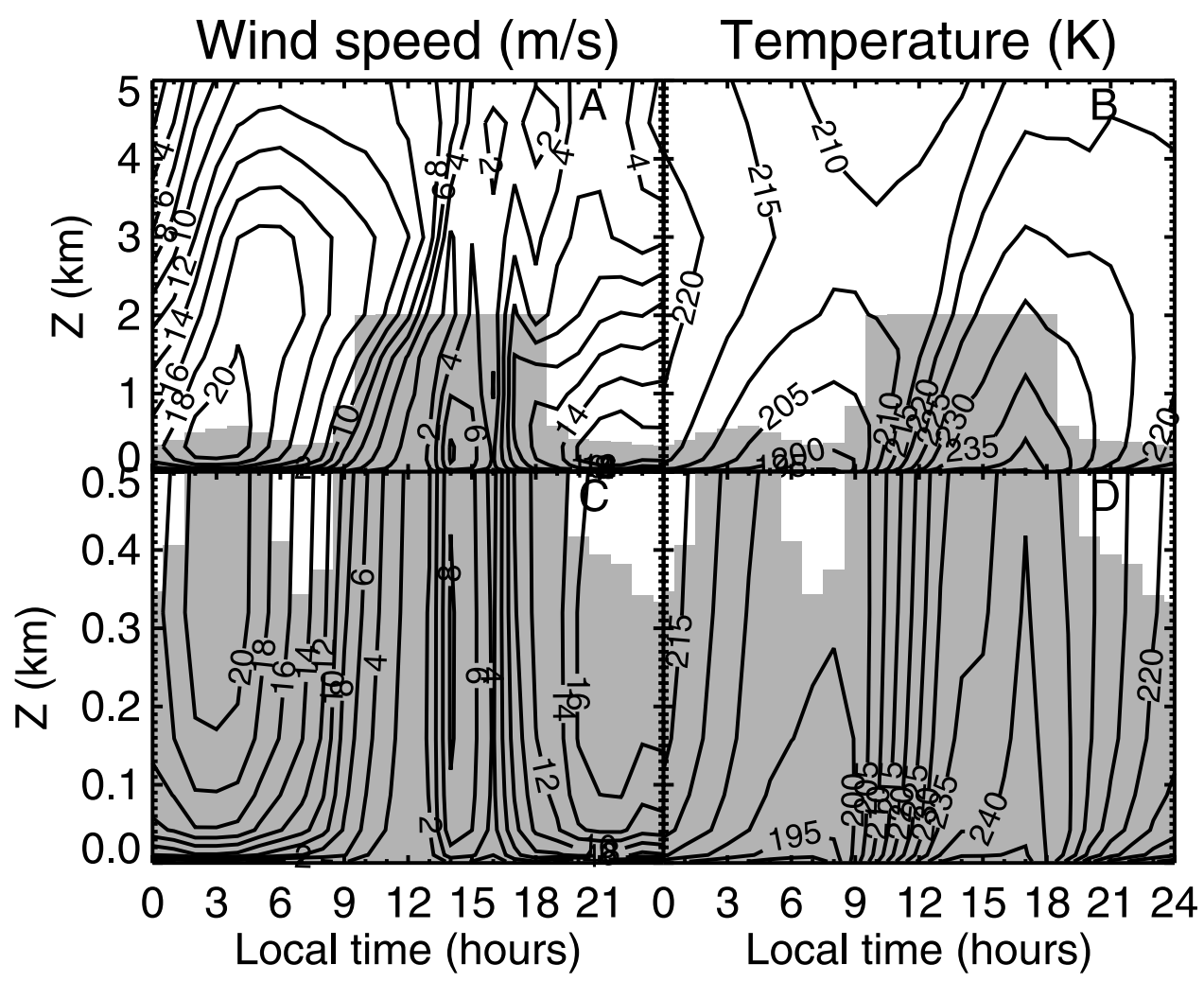

Figure 13. Contour plots of the boundary layer at the Viking Lander 1 site as a function of local time. Figures $13 \mathrm{a}$ and $13 \mathrm{~b}$ are for the range $0-5 \mathrm{~km}$, while Figures $13 \mathrm{c}$ and $13 \mathrm{~d}$ zoom in to the region $0-0.5 \mathrm{~km}$. Figures $13 \mathrm{a}$ and $13 \mathrm{c}$ show wind speed in $\mathrm{m} / \mathrm{s}$ as a function of height and time of day, while Figures $13 \mathrm{~b}$ and $13 \mathrm{~d}$ show air temperature in $\mathrm{K}$. The gray background indicates the model-predicted height of the the planetary boundary layer.

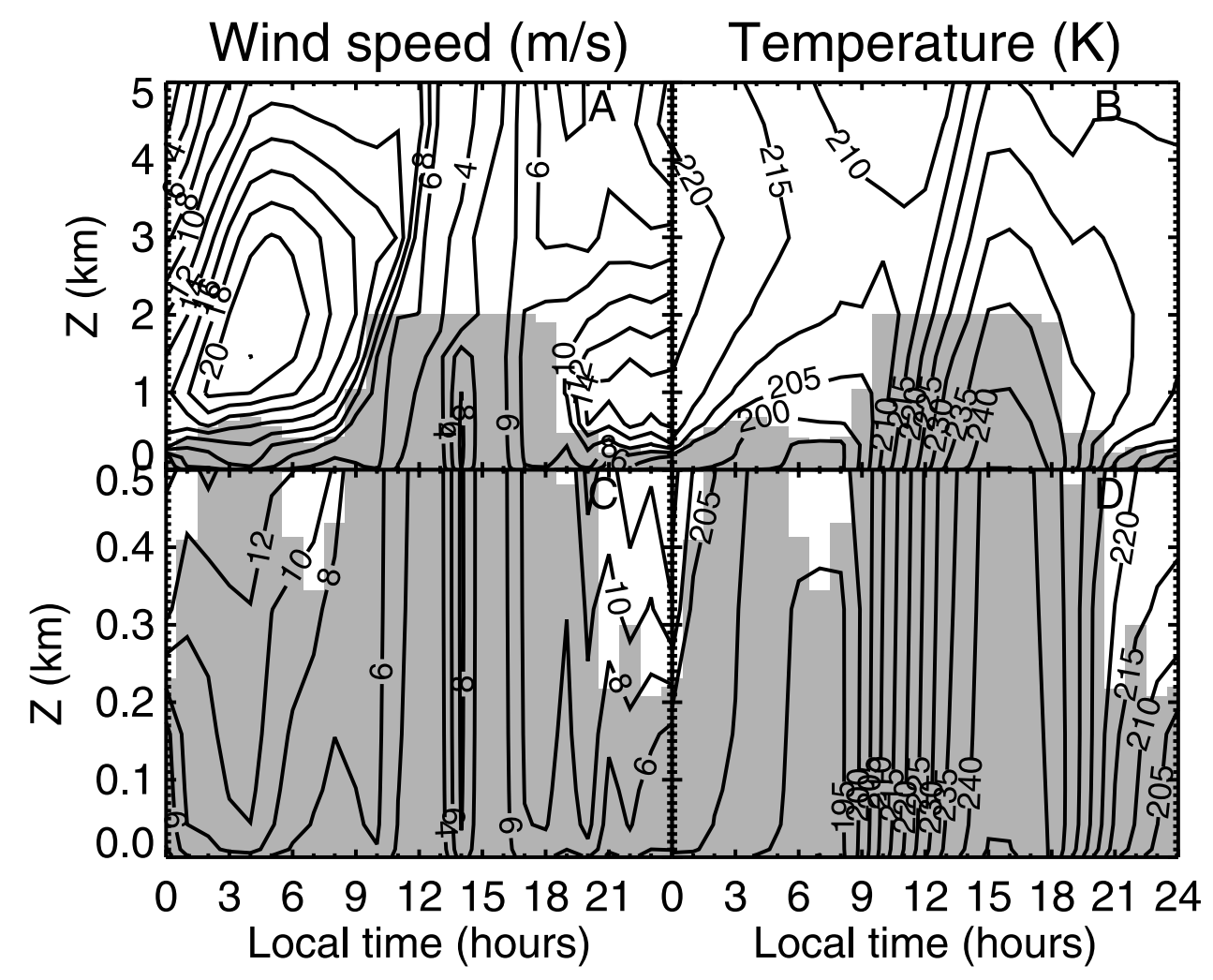

Figure 14. Same as Figure 13, except for using 10 times larger vertical diffusivities. 


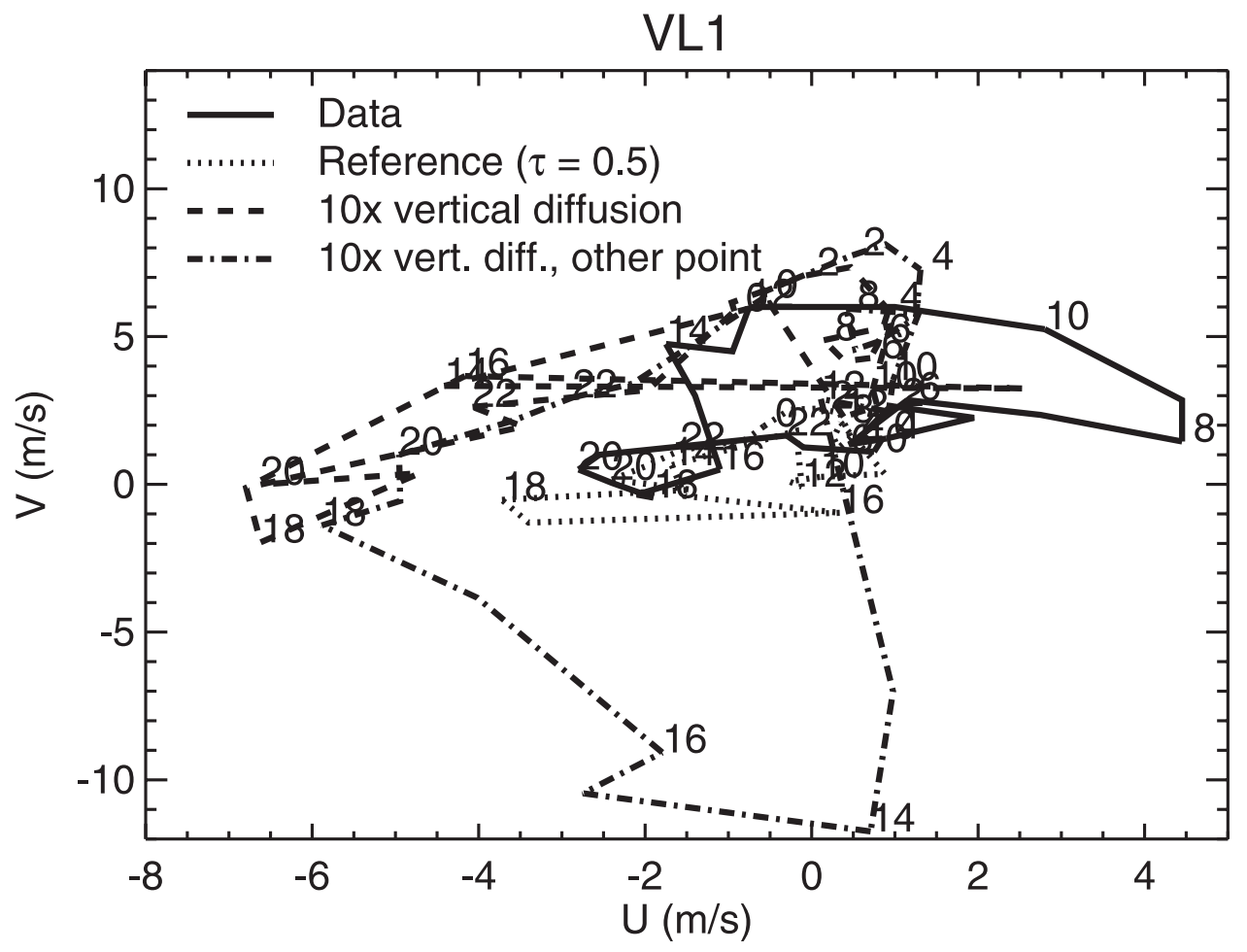

Figure 15. Same as Figure 12, except for the inclusion of velocity vectors from the other sample point mentioned in Figure 11.

topography data set at the reported Viking Lander 1 location. The values used by Haberle et al. [1993] are a slope of 0.003 , downward to the northeast, while the value from the MOLA data is 0.0046 , downward to the southeast. In order to examine the effect of slope, we chose another point in the model domain with a slope direction and magnitude very similar to those used by Haberle et al. [1993]. The wind directions for this point are also plotted in Figure 11, while the hodograph is shown in Figure 15 (labeled in both figures as the "other point"). At this point the model predicts a significantly worse fit to both the wind direction and hodograph of the data. We will further investigate the role of slope on wind velocities while discussing the VL2 data in the next section.

[50] We note that the wind velocities during the Viking Lander 1 and Viking Lander 2 entries simulated by the Mars MM5 (not shown) agree with those simulated by the onedimensional boundary layer model of Haberle et al. [1993]. This means that we also disagree with the magnitude and direction of rotation (with height) of winds derived from entry tracking by Seiff [1993].

\subsection{Viking Lander 2 Site}

[51] At the Viking Lander 2 site we examined two different seasons. The long baseline of observations at Viking Lander 2 affords us the opportunity to examine northern winter conditions that were unavailable at either of the other two previously discussed landing sites. Again on the basis of the availability of data (including compromises in quality among the different variables), we chose to examine the period around $L_{s}=334$. For the northern summer Viking Lander 2 period we chose $L_{s}=130$ in order to compare our simulations to the results reported by Haberle et al. [1993] and Savijärvi and Siili [1993].

\subsubsection{Winter}

[52] Once again, the model simulation of surface pressure demonstrates that the Mars MM5 is accurately propagating the global tidal field generated by the GCM (Figure 16). In this case the semidiurnal tide is well captured, in both magnitude and phase, but the magnitude of the diurnal tide appears to be somewhat underpredicted, although caveats regarding the quality of the data discussed in section 4.2 should be borne in mind.

[53] Near-surface air temperatures are shown in Figure 17. At this season, dust opacity is shown to have a large effect on daytime peak air temperatures. The best fit appears to be for an optical depth of $\sim 1.5$. The model does not appear to fully capture the phasing of the diurnal cycle of air temperature, being somewhat too early to heat up in the morning and too early to cool off at night. Note that this is the opposite behavior to that exhibited at the Mars Pathfinder site. Thus there is no systematic error in the model with respect to lander observations. Instead, discrepancies are landing site specific and therefore more likely related to local errors such as slope, thermal inertia, or albedo. The lander observations for this period are very interesting in that they show a great deal of day-to-day variation in air temperature, on the order of $10 \mathrm{~K}$ or more (not shown). Thus attempts to match the model to the observations are made somewhat difficult. The model does generate day-to-day variation in temperature associated with the passage of baroclinic storm systems generated by the GCM. However, these systems are still quite regular compared to the data. 

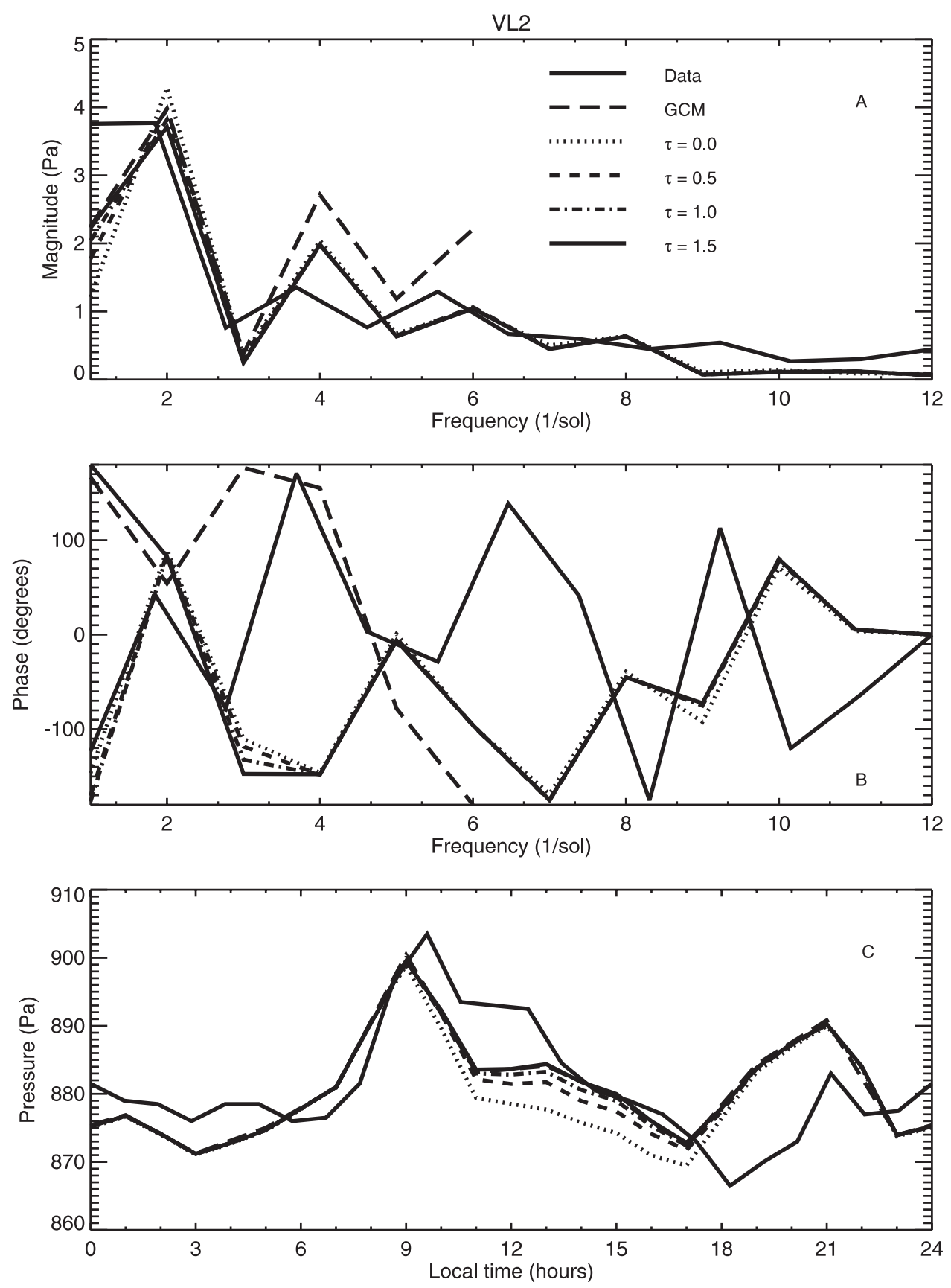

Figure 16. Same as Figure 5, except at the Viking Lander 2 site. The time of year is $L_{s}=334$.

[54] All of the Mars MM5 optical depth cases and the GCM do a good job of fitting the predominantly westerly winds throughout most of the day (Figure 18). Differences occur in the late evening where all of the models suggest a rotation while the data do not rotate. As with the temperature data (as well as the pressure data), the day-to-day variability of the Viking Lander 2 wind data is also quite high. We have attempted to mitigate the variability due to weather by taking an average of two days. However, even after taking an average, errors in simulating the data result from differences in phase of the weather systems and from the failure of the joint model system to generate truly chaotic weather. The fact that the GCM and the Mars MM5 cases agree so well with each other is indicative of the strong control of the wind regime by baroclinic and tidal processes (i.e., there does not appear to be significant modification due to local topography).

[55] Figure 19 shows that the underprediction of wind speeds exhibited at the Viking Lander 1 site during summer also occur at the Viking Lander 2 site during winter. Peak winds during the late afternoon are about a factor of 3 too slow. However, unlike the Viking Lander 1 summer case, the phasing of peak winds is correctly reproduced. In an extension of the experiment with increasing vertical diffusivity, we ran a simulation for this period and location using vertical diffusivities increased by a factor of 10 . The results are shown in Figure 19. This simulation has significantly increased winds which now reach roughly $75 \%$ of the observed peak winds. 


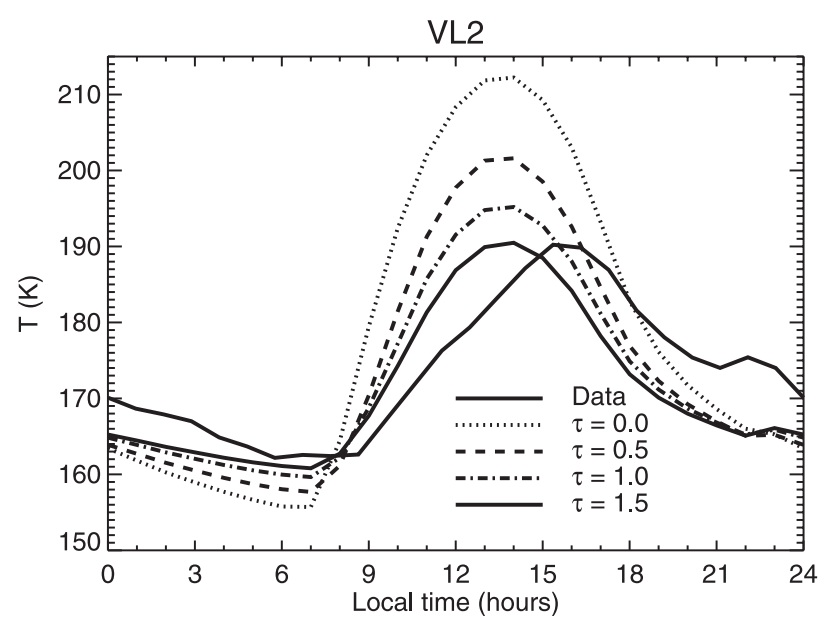

Figure 17. Plots of the diurnal temperature cycle at the Viking Lander 2 site during northern winter for both the measured data and the model output for various optical depth cases.

[56] Increasing the vertical diffusivity effectively couples the lower levels of the model, equivalent to those sampled by the Viking Lander sensors, to the stronger upper level winds. An equivalent increase in vertical diffusivity occurs in the standard model as the optical depth is decreased. Here, the reduction in optical depth cools the atmosphere and warms the surface. The effect of this is to decrease the static stability, increasing the vertical mixing. Figure 20 shows the hodographs for the four optical depth cases and demonstrates that the strongest daytime winds occur for the clearest atmosphere case and decrease monotonically with increasing optical depth.

\subsubsection{Summer}

[57] We decided to run a second simulation at Viking Lander 2 during northern summer $\left(L_{s}=130\right)$ primarily to compare to previous studies [Haberle et al., 1993; Savijärvi and Siili, 1993]. In this case we choose not to show the different optical depth cases, since this type of experiment was already done at the same location (in winter) and during

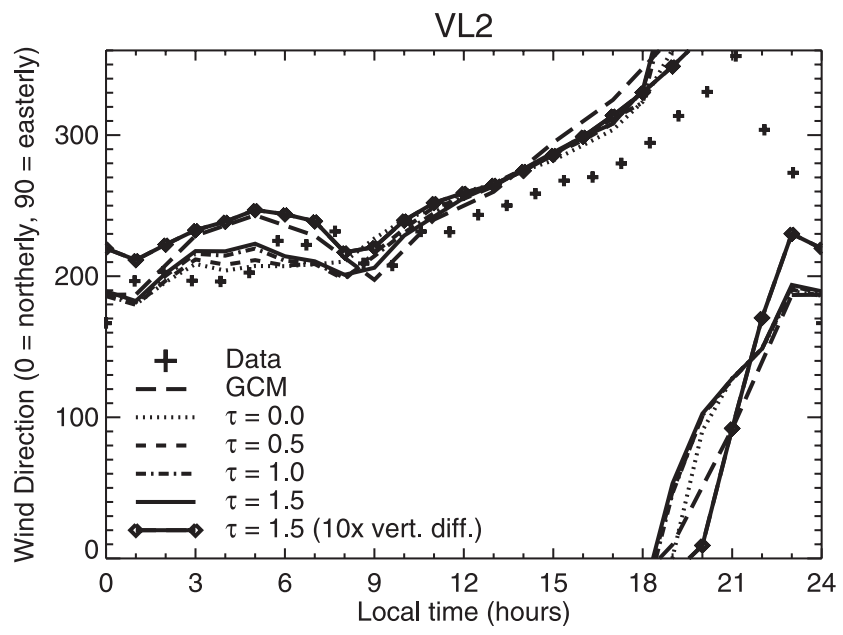

Figure 18. Same as Figure 8, except at the Viking Lander 2 site. The time of year is $L_{s}=334$.

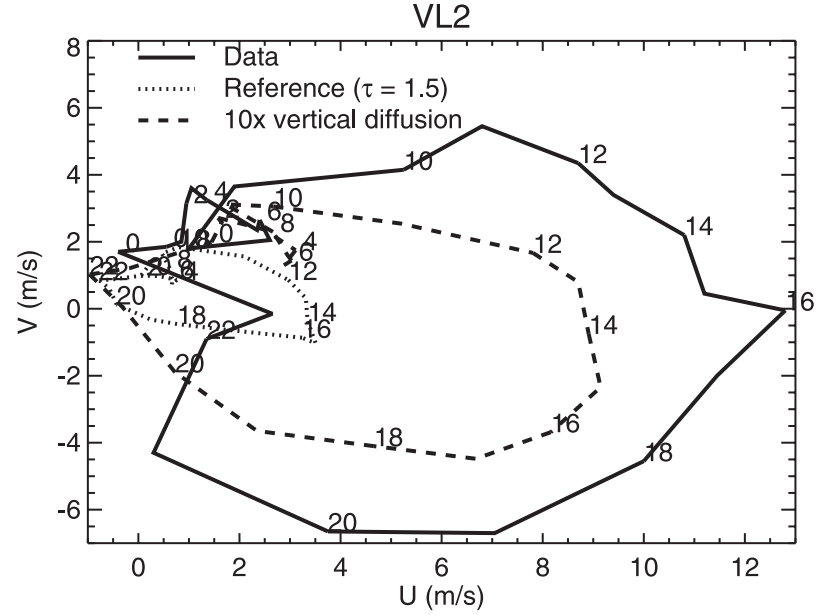

Figure 19. Hodograph of wind velocities at the Viking Lander 2 site during northern winter. The standard case is for an optical depth of 1.5 .

the same period (at Viking Lander 1). We have chosen instead to show the best fit optical depth, 0.5 , and then vary other parameters to investigate their effect. It should also be noted that this reference case includes the effect of 10 times vertical diffusivity.

[58] We do not show any pressure comparisons for the summer period $\left(L_{s}=130\right)$ as the data is so poor. The temperature data are quite good and are shown in Figure 21. The model fits to data for this period are reasonable, and only the best fit opacity is shown. Slight differences between the model and data include underprediction of nighttime temperatures and slightly delayed cooling in the evening. These differences (which were similar in the winter case) suggest that the thermal inertia used in the model is too low or there are slope influences on the phasing of absorbed solar radiation.

[59] Wind directions are shown in Figure 22. The Mars MM5 does a reasonable job fitting directions from late afternoon until late morning. During the middle of the day

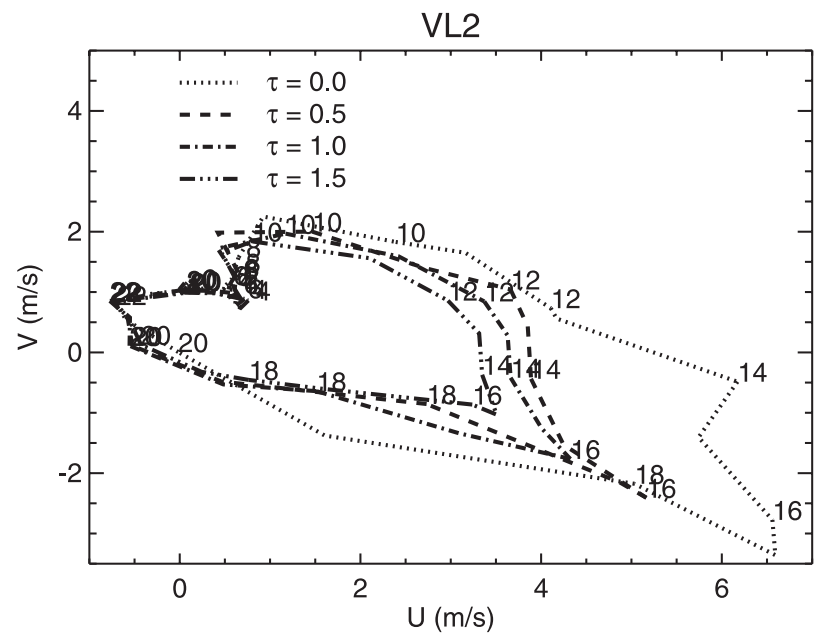

Figure 20. Hodograph of wind velocities at the Viking Lander 2 site during northern winter for the various optical depth cases. 


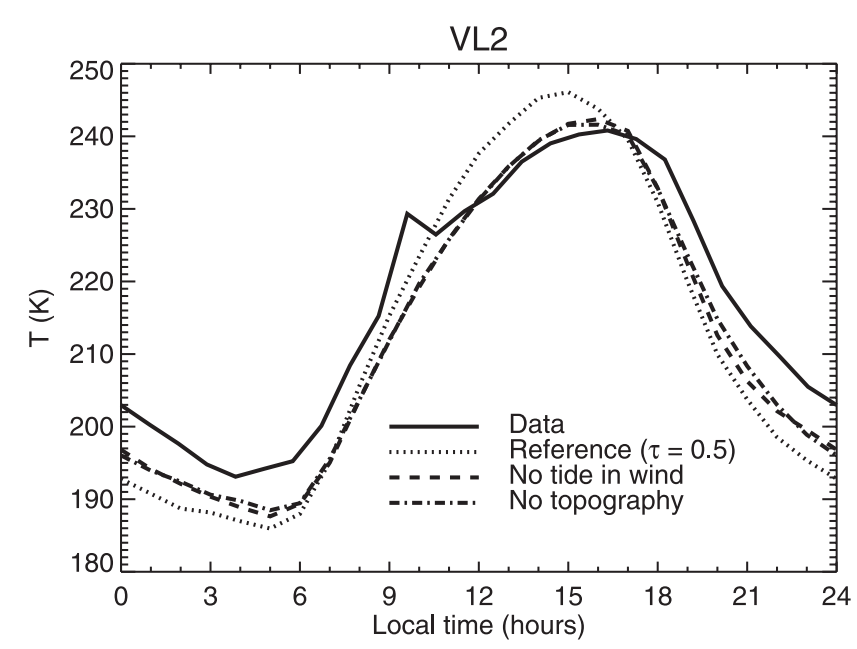

Figure 21. Diurnal cycle of near-surface air temperatures, from measurements and model simulations, at the Viking Lander 2 site during northern summer. The reference case is for an optical depth of 0.5 and a 10 times increase in verical diffusivity (see text). "No tide in wind" refers to the simulation where the daily average wind was used for the boundary conditions and was invariant in time. "No topography" refers to the simulation where the model domain was initialized with a flat surface at the height of the Viking Lander 2 location.

the reference case oscillates in direction somewhat more than the data, but in the same sense. This can be seen in Figure 23, where the wind magnitudes are comparable to those of the data. Unfortunately, the phasing of wind maxima does not correspond to the data. The model generates peak winds during the late afternoon and early evening, while the data suggest peak winds in the mid to late morning. This again differs from studies using simple, one-dimensional slope models [Haberle et al., 1993; Savijärvi and Siili, 1993]. We thus experimented with the effects of slope versus global tide on the wind directions.

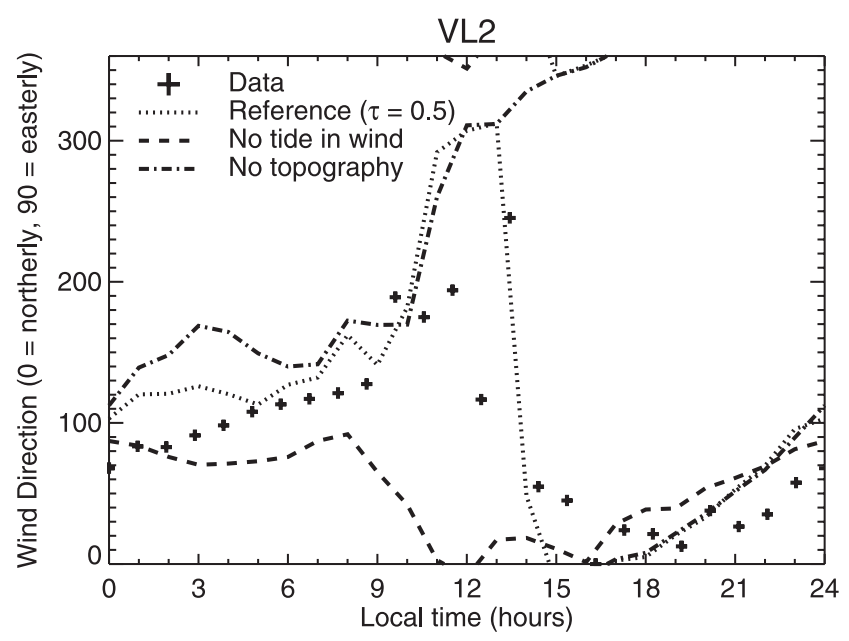

Figure 22. Wind directions as a function of local time for the Viking Lander 2 location during northern summer. The different cases are the same as described in Figure 21.

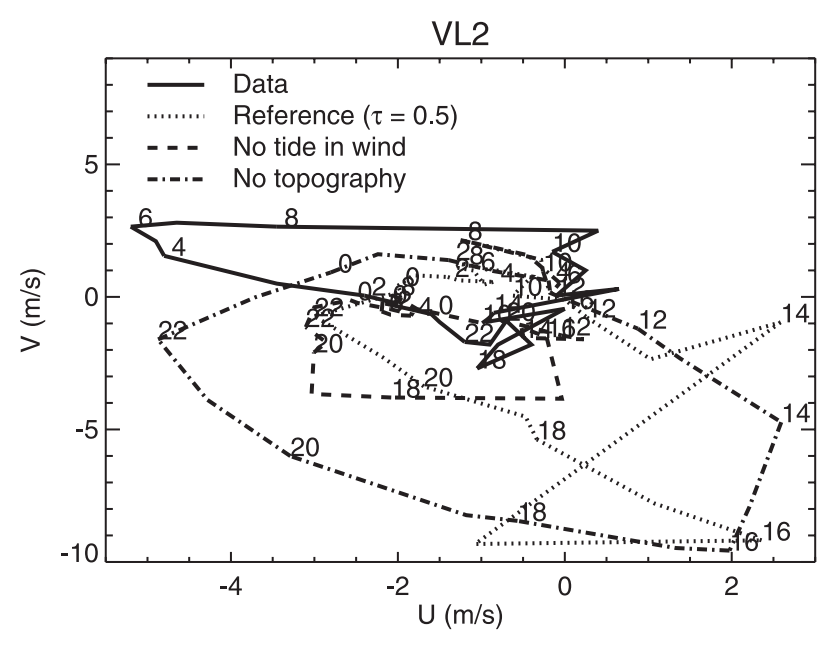

Figure 23. Hodograph of the wind velocity vectors for the measured data and for the model simulations at the Viking Lander 2 site during northern summer. The different cases are the same as described in Figure 21.

[60] In the first modified simulation we removed the effects of the global tide on the imposed wind field (i.e., the boundary and initial conditions). This is equivalent to the imposition of a uniform wind in the slope models. The results are shown in Figures 22 and 23. The quality of the fit to wind direction is significantly degraded. The directions begin to disagree just after midnight and do not agree again until late evening. Further, there is very little variation in direction $\left(<90^{\circ}\right)$ during the entire day. This can also be seen in the hodograph. Although the conditions now mimic those used in slope wind models, the Mars MM5 does not generate the observed velocities.

[61] We proceeded to remove the topography to investigate the effect of global tides in isolation from slope effects. These results can also be seen in Figures 22 and 23. The fit to directions again is relatively poor, except in the late afternoon and evening. However, in this case a full $360^{\circ}$ rotation occurs, and the simulation is somewhat similar to the standard simulation for the first half of the day. The hodograph for this case is quite simple, with a smooth circular rotation, peak winds occurring in the late afternoon and exceeding the peak observed values by about a factor of 2 .

[62] The combination of these results suggests that both tides and slopes contribute. This is also supported by the Viking Lander 1 result where a different location within the model domain with a different slope generated a rather different hodograph than the standard simulation (Figure 15). Thus we repeated this experiment with the Viking Lander 2 summer simulation. We explicitly selected three more locations within the model domain with slope directions that were $\sim 90^{\circ}$ apart. The Viking Lander 2 site within this domain has a slope of 0.006 , downward to the northwest. We chose locations near the center of the domain with slopes of (point 2) 0.011, downward to the southeast; (point 3) 0.011, downward to the southwest; and (point 4) 0.003 , downward to the northeast. In this case the hodographs appear largely insensitive to local slope direction, as can be seen in Figure 24. This is consistent with examination of time series maps of wind direction, where the direction is fairly uniform across the entire domain $(\sim 120$ 


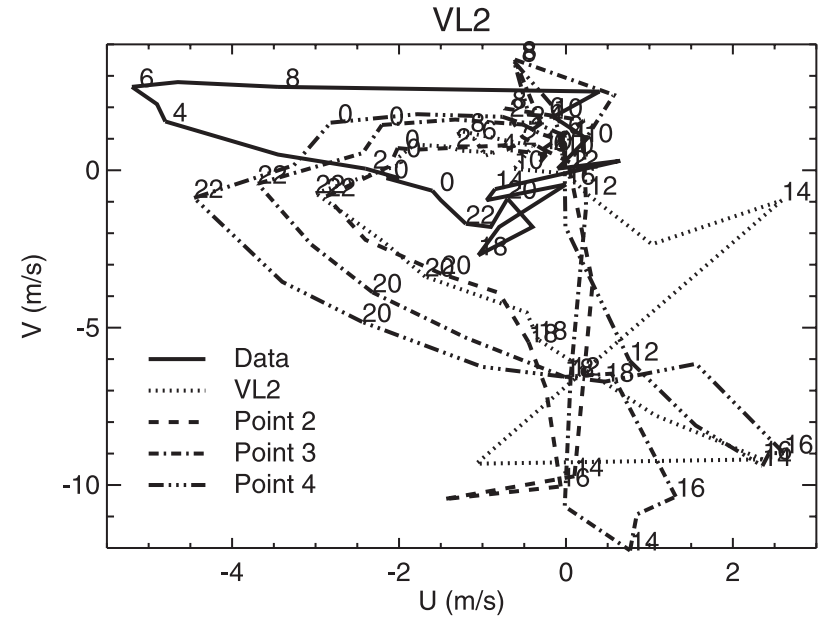

Figure 24. Hodograph of the wind velocity vectors from the reference model simulation for four different locations in the model domain, plotted along with the wind velocities measured at Viking Lander 2 during northern summer. The four different points have slopes about $90^{\circ}$ from each other, and magnitude of the largest slope is within a factor of 4 of the smallest.

$\mathrm{km}$; not shown). This is again different from the case of Viking Lander 1. Apparently, local slopes (on the scale of a few to a few tens of kilometers) are not particularly important in determining wind direction at the Viking Lander 2 site. The finding that the elimination of slope does affect wind direction suggests the important scale of slope forcing is between the scale resolvable by the GCM and that of the mesoscale model domain.

[63] Although not shown, we also examined the effect of horizontal resolution on the quality of fits at the Viking Lander 2 site. We conducted experiments with a horizontal resolution of $4 \mathrm{~km}$ (our default for all of the previous lander simulations), $16 \mathrm{~km}$, and $60 \mathrm{~km}$ (in each case with a 32 by 32 grid). There was no noticeable difference between the simulations, in any of the variables, which is consistent with the results described in the previous paragraph. That topography of a scale smaller than roughly $100 \mathrm{~km}$ does not markedly influence the simulations is not a general result, as indicated by the Viking Lander 1 simulations. The landing sites were preselected to be relatively flat, and this appears to be particularly the case at Viking Lander 2. However, in regions where topography is large on small horizontal scales (e.g., craters, canyons, channels, chaotic terrain, etc.), the circulation is expected to exhibit sensitivity.

\section{Summary}

[64] We have taken the Earth PSU/NCAR Mesoscale Model Version 5 (MM5) and fully converted it to Mars using the Mars-specific parameterizations of the GFDL Mars GCM. We use output from the GFDL Mars GCM to initialize and drive (i.e., provide time-evolving boundary conditions for) the Mars MM5.

[65] In comparing to the GCM, the Mars MM5 is found to accurately capture most of the structures generated in the GCM when the Mars MM5 domain is essentially global. This fidelity extends even to the reasonable simulation of the three-dimensional distribution of dust, which involves detailed radiative and dynamical feedback systems. We find the biggest limitation to be imposed by the finite model height and the rigid lid. This has the effect in the global simulations of confining the Hadley circulation. As a result, the zonal wind field and the distribution of dust particles are modified from those in the GCM. This suggests that careful attention must be paid to the design of mesoscale model domains for experiments where simulation of the Hadley flow is important, and a sufficiently high model top must be selected. For simulations of near-surface flow phenomena this is less important as near-surface flow on Mars appears to be strongly controlled by topography. Some attention must also be given to the horizontal extent of the domain and the location and scale of the processes to be examined, for example, making sure that the domain has sufficient latitudinal extent to fully capture the width of baroclinic storm systems.

[66] Near-surface air temperatures measured by the two Viking Landers and Mars Pathfinder are relatively wellsimulated for all seasons examined. This suggests that both the subsurface heat diffusion code and the surface layer parameterization are good. Some errors in phasing of the diurnal temperature cycle are found, especially at Viking Lander 2 during winter. These errors suggest either small errors in the thermal inertia used or the lack of treatment of slopes in the calculation of absorbed insolation.

[67] The Mars MM5 faithfully reproduces the variations in surface pressure generated by the GCM. As most of these pressure variations result from large- to global-scale dynamical systems (e.g., the global tide or baroclinic storm systems), it is not surprising that the Mars MM5 does not significantly alter them. Indeed, the fact that they are reproduced so faithfully suggests that the coupling of the Mars MM5 to the GCM through the time-evolving boundary conditions is well-implemented.

[68] Wind directions for all the landing sites and for all seasons are relatively well-reproduced. In most cases the Mars MM5 variation in wind directions is not greatly different from that generated by the GCM. This suggests that control of wind directions is provided by the global tide as modified by topography on a scale greater than a few hundred kilometers. However, we have observed that locations in the Mars MM5 model domain that are more proximate to large local topography exhibit significant deviations from the large-scale (GCM-predicted) flow (not shown). We also note that the wind directions provided by the GCM are reported for a height of roughly $200 \mathrm{~m}$ above the surface and that, consequently, there appears to be little rotation in the lower boundary layer. In general, the prediction of wind directions appears to be quite good.

[69] Unfortunately, we systematically underpredict the peak wind speeds at all locations and all seasons. Furthermore, the phasing of wind speed as a function of local time is not well-reproduced for any landing site, except for Viking Lander 2 in winter. In order to generate daily variations in wind speed comparable to those observed, we increased the vertical diffusivity by a factor of 10 . However, this increase did not correct the phasing problem. In addition, we have no physical justification for the increase, and therefore further work needs to be done to examine the behavior of wind in the lower boundary layer. Specifically, a detailed study of the applicability of the terrestrial planetary boundary layer 
parameterizations to Mars and their use in Martian numerical models needs to be undertaken. However, such a large study is beyond the scope of this paper. Given that we underpredict wind speeds, and that the wind speeds and their phasing at $\sim 200 \mathrm{~m}$ agree with the GCM, the Mars MM5 represents a conservative tool for the investigation of processes such as dust lifting, where wind speed is important. It should be noted that wind speeds generated by the mesoscale model near the 5 $\mathrm{m}$ level for the Mars Pathfinder site and season agree quite well with those generated by the NASA Ames Mars GCM [Haberle et al., 1999], which includes a "level 2" Mellor and Yamada [1982] scheme. Thus there would not appear to be a major difference between these two model boundary layer schemes. The one-dimensional boundary layer model of Haberle et al. [1993] is able to fit the observed wind speeds despite neglecting what this study suggests should be important dynamics, that is, global tides. However, the fits result from tuning the slope magnitude and direction and a mixing depth parameter. The two-dimensional model of Savijärvi and Siili [1993] does not produce significantly better fits to the landing site winds than the Mars MM5. Clearly, we do not yet understand the mix of processes controlling surface level winds.

[70] A significant result in relation to previous studies of the diurnal cycle of winds relates to their driving mechanism. In one-dimensional boundary layer models it has been common to apply uniform upper level winds and allow the diurnal cycle of wind to be generated by slope forcing [Haberle et al., 1993; Savijärvi and Siili, 1993]. Our results suggest that the global tide is at least as important as local slope in generating the variability of winds. Indeed, at Mars Pathfinder and Viking Lander 2, slopes on a scale smaller than that of the GCM grid spacing (a few hundred kilometers) are not particularly important. Future work needs to be done focusing on wind speed phasing that will require a detailed study of the tides generated in the GCM and passed to the Mars MM5, the interaction of these tides with topography, and the sensitivity of the tides to the three-dimensional distribution of dust. The importance of sub-GCM-scale topography in generating slope winds which interact with the global tidal systems requires that such a study be undertaken with a joint GCM/Mars MM5 modeling system.

[71] The work undertaken within this study suggests that when used in combination with a GCM, and when attention is paid to the design of the Mars MM5 experiments and model domain, the Mars MM5 promises to be a powerful tool for the investigation of processes central to the Martian climate on scales from hundreds of kilometers to tens of meters.

[72] Acknowledgments. We would like to thank R. John Wilson for extensive assistance, discussion, and inspiration for this project. Without his help, it would not have been possible. We would further like to thank Andrew P. Ingersoll and Ashwin R. Vasavada for numerous discussions and advice. This project also benefited from the comments and assistance of Arden Albee, Bruce Murray, and Yuk Yung. We would also like to thank Tim Schofield for provision of the Pathfinder wind data prior to its PDS release. Finally, we thank an anonymous reviewer for useful comments and suggestions.

\section{References}

Andrews, D. G., J. D. Mahlman, and R. W. Sinclair, Eliassen-Palm diagnostics of wave-mean flow interaction in the GFDL "SKYHI" general circulation model, J. Atmos. Sci., 40, 2768-2784, 1983.
Anthes, R. A., and T. Warner, Development of hydrodynamic models suitable for air pollution and other mesometeorological studies, Mon. Weather Rev., 106, 1045-1078, 1978.

Arakawa, A., and V. R. Lamb, Computational design of the basic dynamical processes of the UCLA general circulation model, Methods Computat. Phys., 17, 173-265, 1977.

Briegleb, B. P., Delta-Eddington approximation for solar radiation in the NCAR community climate model, J. Geophys. Res., 97, 7603-7612, 1992.

Burk, S. D., Diurnal winds near the Martian polar caps, J. Atmos. Sci., 33, 923-939, 1976

Dudhia, J., A nonhydrostatic version of the Penn State-NCAR mesoscale model: Validation tests and simulation of an Atlantic cyclone and cold front, Mon. Weather Rev., 121, 1493-1513, 1993.

Haberle, R. M., and B. M. Jakosky, Atmospheric effects on the remote determination of thermal inertia on Mars, Icarus, 90, 187-204, 1991.

Haberle, R. M., C. B. Leovy, and J. B. Pollack, Some effects of global dust storms on the atmospheric circulation of Mars, Icarus, 50, 322-367, 1982.

Haberle, R. M., H. C. Houben, R. Hertenstein, and T. Herdtle, A boundary layer model for Mars: Comparison with Viking Lander and entry data, $J$. Atmos. Sci., 50, 1544-1559, 1993.

Haberle, R. M., et al., General circulation model simulations of the Mars Pathfinder atmospheric structure investigation/meterology data, J. Geophys. Res., 104, 8957-8974, 1999.

Hamilton, K., R. J. Wilson, J. D. Mahlman, and L. J. Umscheid, Climatology of the SKYHI troposphere-stratosphere-mesosphere general circulation model, J. Atmos. Sci., 52, 5-43, 1995.

Hong, S.-Y., and H.-L. Pan, Nonlocal boundary layer vertical diffusion in a medium-range forecast model, Mon. Weather Rev., 124, 2322-2339, 1996.

Hourdin, F., A new representation of the $\mathrm{CO}_{2}$ 15-micron band for a Martian general circulation model, J. Geophys. Res., 97, 18,319-18,335, 1992.

Jacobson, M. Z., Fundamentals of Atmospheric Modeling, Cambridge Univ. Press, New York, 1999

Joshi, M., R. Haberle, J. Barnes, J. Murphy, and J. Schaeffer, Low-level jets in the NASA Ames Mars general circulation model, J. Geophys. Res., 102, 6511-6524, 1997.

Kirk, R. L., et al., Digital photogrammetric analysis of the IMP camera images: Mapping the Mars Pathfinder landing site in three dimensions, $J$. Geophys. Res., 104, 8869-8888, 1999.

Mellor, G. L., and T. Yamada, Development of a turbulence closure model for geophysical fluid problems, Rev. Geophys., 20, 851-875, 1982.

Murphy, J. R., J. B. Pollack, R. M. Haberle, C. B. Leovy, O. B. Toon, and J. Schaeffer, Three-dimensional numerical simulation of Martian global dust storms, J. Geophys. Res., 100, 26,357-26,376, 1995.

Paige, D. A., and K. D. Keegan, Thermal and albedo mapping of the polar regions of Mars using Viking thermal mapper observations, 2, South polar region, J. Geophys. Res., 99, 25,993-26,013, 1994.

Paige, D. A., J. E. Bachman, and K. D. Keegan, Thermal and albedo mapping of the polar regions of Mars using Viking thermal mapper observations, 1, North polar region, J. Geophys. Res., 99, 25,95925,991, 1994.

Palluconi, F. D., and H. H. Kieffer, Thermal inertia mapping of Mars for $60^{\circ} \mathrm{S}$ to $60^{\circ} \mathrm{N}$, Icarus, $45,415-426,1981$.

Pleskot, L. K., and E. D. Miner, Time variability of Martian bolometric albedo, Icarus, 45, 179-201, 1981.

Pollack, J. B., R. M. Haberle, J. R. Murphy, J. Schaeffer, and H. Lee, Simulations of the general-circulation of the Martian atmosphere, 2, Seasonal pressure variations, J. Geophys. Res., 98, 3149-3181, 1993.

Richardson, M. I., A general circulation model study of the Mars water cycle, Ph.D. thesis, Univ. of Calif., Los Angeles, 1999.

Savijärvi, H., and T. Siili, The Martian slope winds and the nocturnal PBL jet, J. Atmos. Sci., 50, 77-88, 1993.

Schofield, J. T., J. R. Barnes, D. Crisp, R. M. Haberle, S. Larsen, J. A. Magalhães, J. R. Murphy, A. Seiff, and G. Wilson, The Mars Pathfinder Atmospheric Structure Investigation/Meteorology (ASI/MET) Experiment, Science, 278, 1752-1758, 1997.

Seiff, A., Mars atmospheric winds indicated by motion of the Viking landers during parachute descent, J. Geophys. Res., 98, 7461-7474, 1993.

Smith, P. H., and M. Lemmon, Opacity of the Martian atmosphere measured by the Imager for Mars Pathfinder, J. Geophys. Res., 104, 89758985, 1999.

Toigo, A. D., M. I. Richardson, R. J. Wilson, H. Wang, and A. P. Ingersoll, A first look at dust lifting and dust storms near the south pole of Mars with a mesoscale model, J. Geophys. Res., 107, 10.1029/2001JE001592, in press, 2002.

Troen, I. B., and L. Mahrt, A simple model of the atmospheric boundary layer: Sensitivity to surface evaporation, Boundary Layer Meteorol., 37 , $129-148,1986$. 
Vasavada, A. R., J.-P. Williams, D. A. Paige, K. E. Herkenhoff, N. T. Bridges, R. Greeley, B. C. Murray, D. S. Bass, and K. S. McBride, Surface properties of Mars' polar layered deposits and polar landing sites, J. Geophys. Res., 105, 6961-6970, 2000.

Wilson, G. R., and M. Joshi, The Martian surface boundary layer, in The Fifth International Conference on Mars [CD-ROM], abstract 6221, LPI Contrib. 972, Lunar and Planet. Inst., Houston, Tex., 1999.

Wilson, R. J., A general ciculation model simulation of the Martian polar warming, Geophys. Res. Lett., 24, 123-126, 1997.

Wilson, R. J., and K. Hamilton, Comprehensive model simulation of thermal tides in the Martian atmosphere, J. Atmos. Sci., 53, 1290-1326, 1996.

Wilson, R. J., and M. I. Richardson, The Martian atmosphere during the Viking Mission, 1, Infrared measurements of atmospheric temperatures revisited, Icarus, 145, 555-579, 2000.
Zurek, R. W., J. R. Barnes, R. M. Haberle, J. B. Pollack, J. E. Tillman, and C. B. Leovy, Dynamics of the atmosphere of Mars, in Mars, edited by H. H. Kieffer et al., pp. 835-933, Univ. of Ariz. Press, Tucson, 1992.

M. I. Richardson, Division of Geological and Planetary Sciences, MS 150-21, California Institute of Technology, Pasadena, CA 91125, USA. (mir@gps.caltech.edu)

A. D. Toigo, Center for Radiophysics and Space Research, 326 Space Sciences Building, Cornell University, Ithaca, NY 14853, USA. (toigo@ gps.caltech.edu) 
A

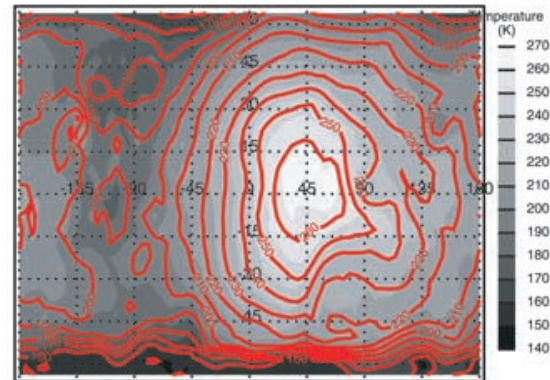

C

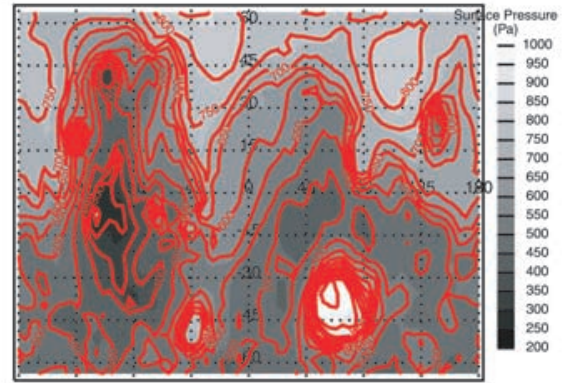

E

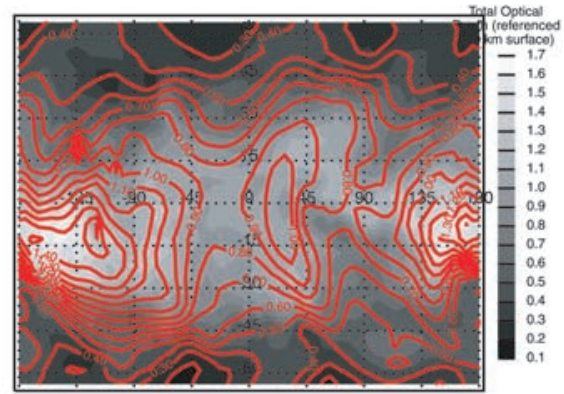

G

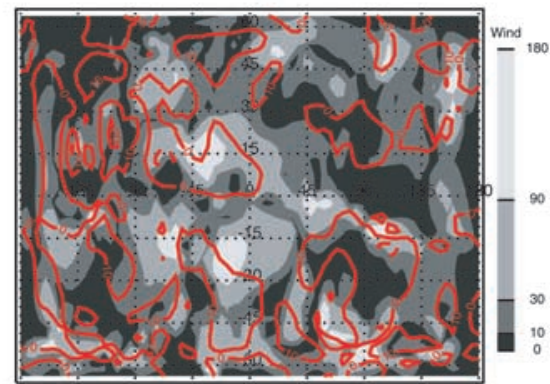

B

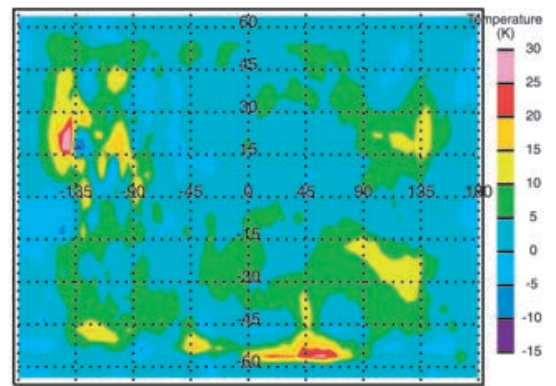

D

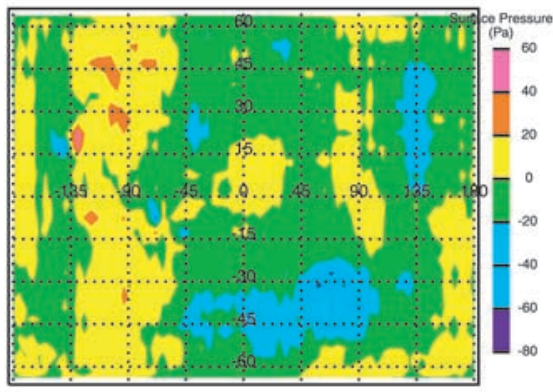

F

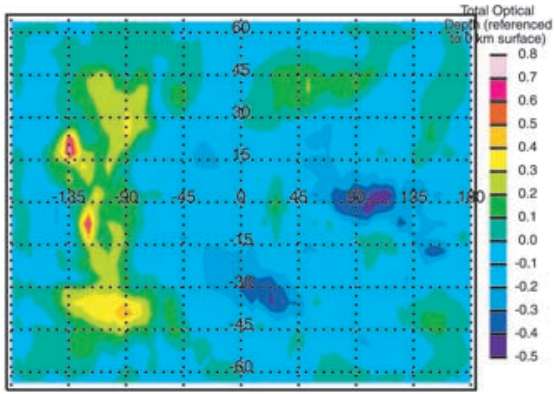

$\mathrm{H}$

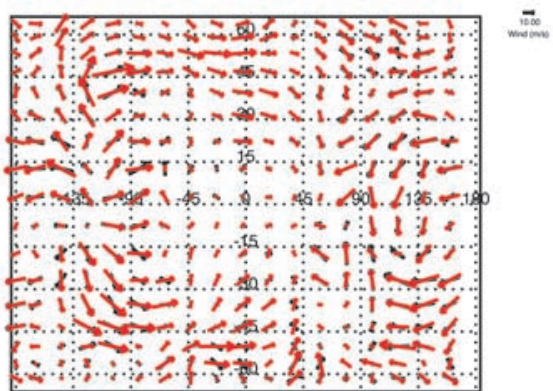

Figure 1. Map projections of model output for various variables at $L_{s}=180$. The local time is noon at $0^{\circ}$ longitude. For the upper six plots, the left-hand column is GCM and Mars MM5 output plotted on top of each other, with the GCM output in the background as a gray shading and the Mars MM5 overplotted as contours. The right-hand column is the difference of the output, Mars MM5 output minus GCM output. (a and b) Temperature. (c and d) Surface pressure. (e and f) Total visible optical depth (referenced to the $0 \mathrm{~km}$ surface). (g) The absolute difference in wind direction, in degrees, is plotted in the background as a gray shading, with ranges labeled by the scale bar at the right. The red contours represent the difference in wind speed between the two models (Mars MM5 minus GCM). (h) Wind vectors for the GCM (plotted in black) and the Mars MM5 (plotted in red). Scale bar for wind speed is at the upper right. 
A
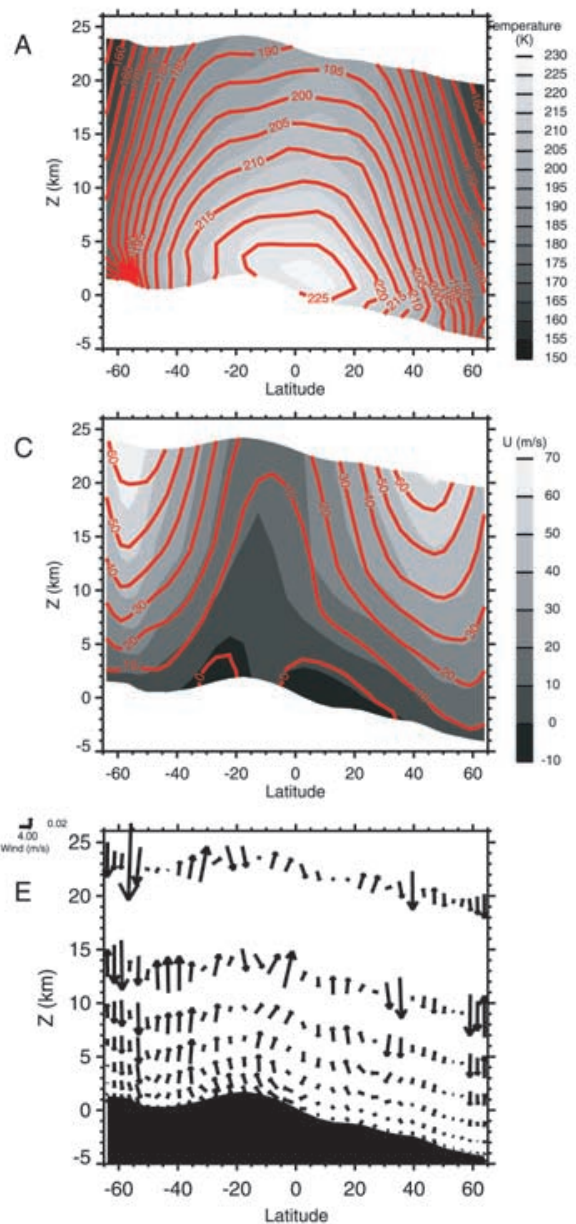

G

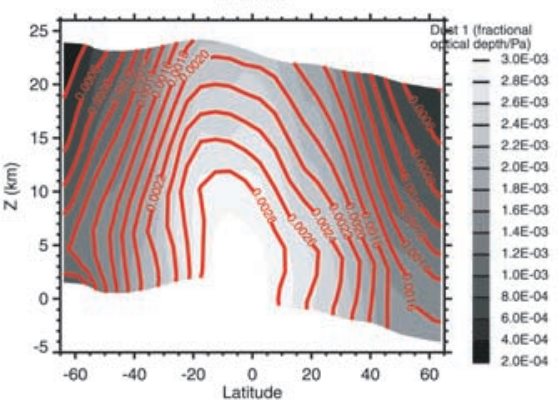

B
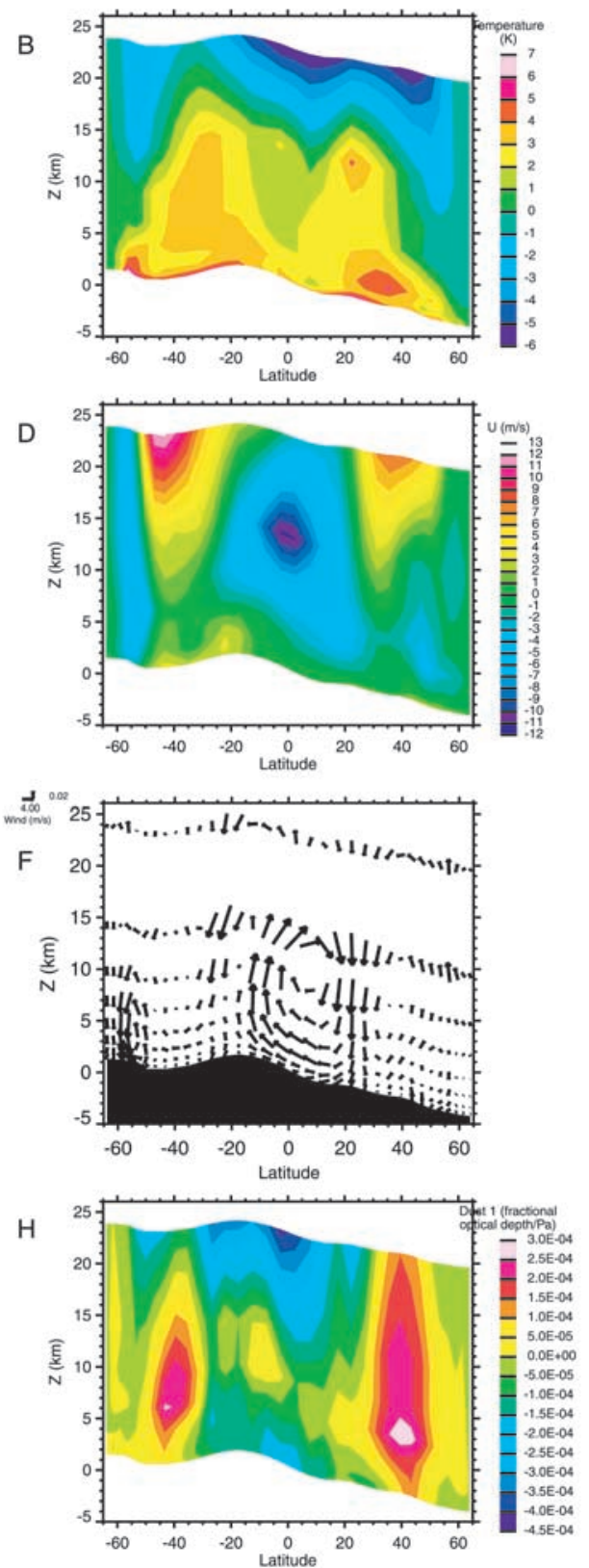

Figure 2. Zonal averages of model output, plotted as latitude versus height, for $L_{s}=180$. Except for the wind vector figures, the left-hand column represents GCM data in the background as a gray shading with Mars MM5 data overplotted in red contours, and the right-hand column is the difference between the two models, Mars MM5 minus GCM. (a and b) Temperature. (c and d) Zonal wind. (e and f) Mean meridional circulation. The vertical velocities have been exaggerated by a factor of 200, and appropriate vector scale bars are at the upper left of the figures. (e) GCM. (f) Mars MM5. (g and h) Dust amount. The units of dust used here are fractional optical depth over the grid box horizontal area per unit thickness of the grid box in pressure. 
A

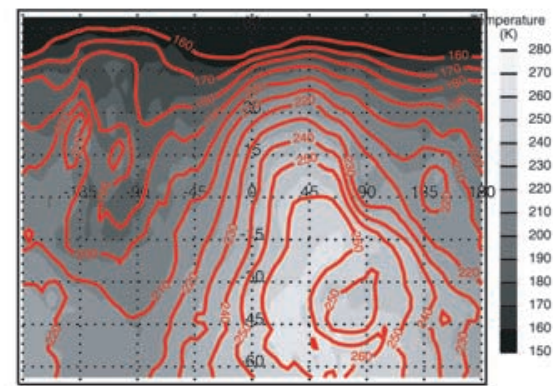

C

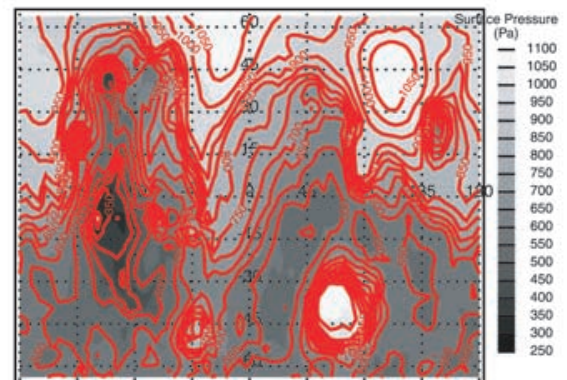

$\mathrm{E}$

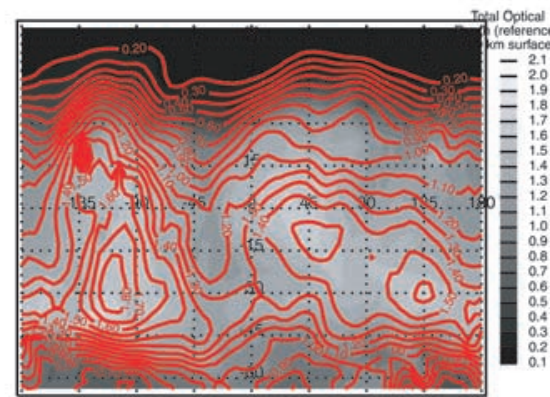

G

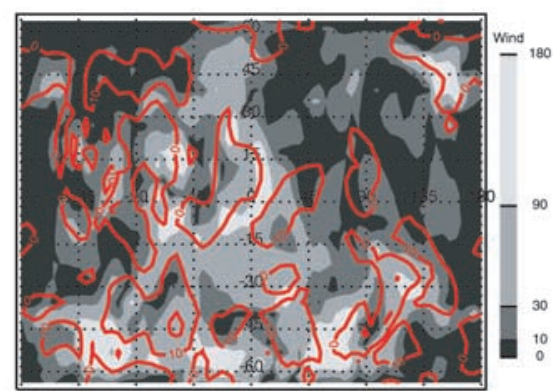

B

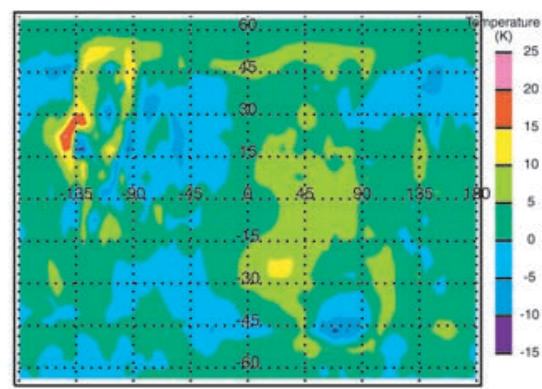

D

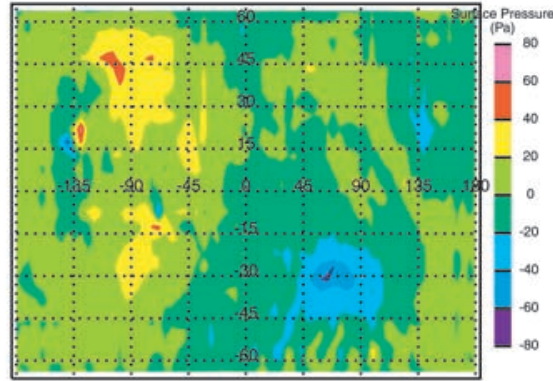

$\mathrm{F}$

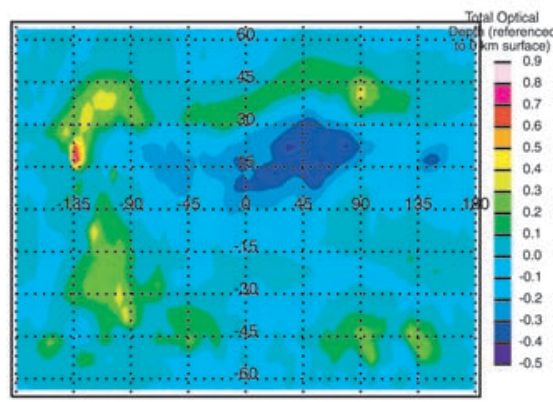

$\mathrm{H}$

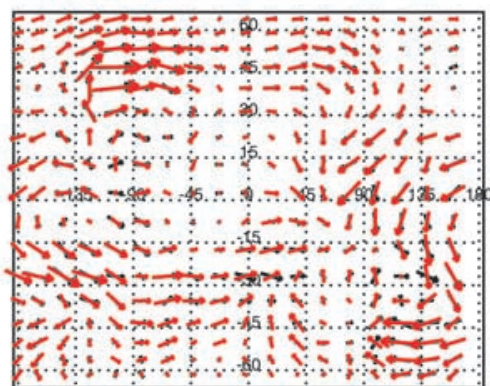

Figure 3. Same as Figure 1, except for $L_{s}=270$. 
A

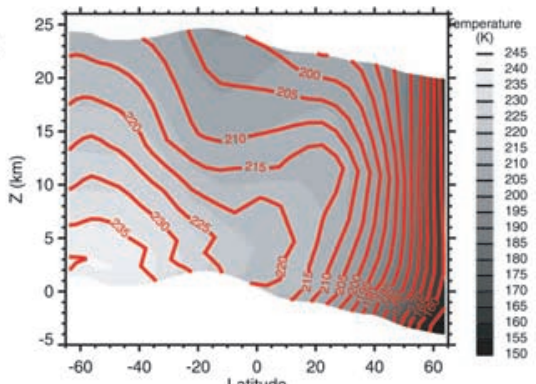

C
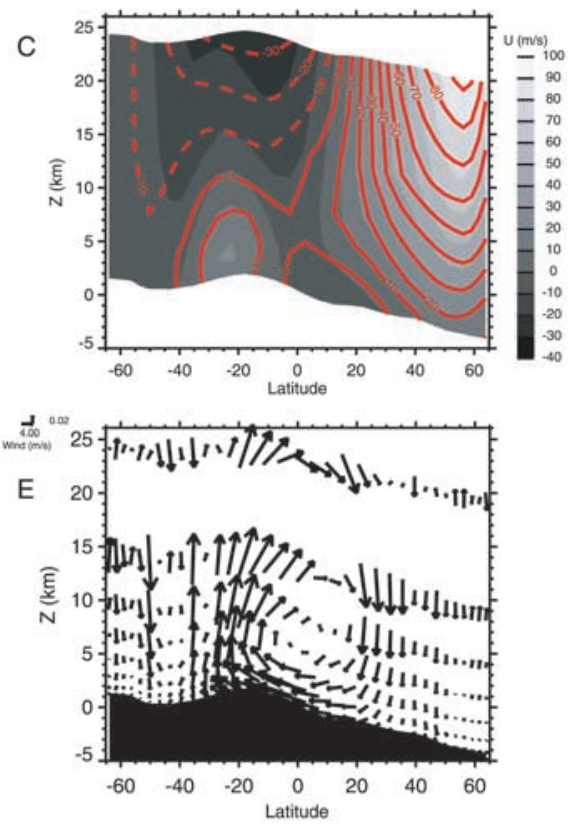

G

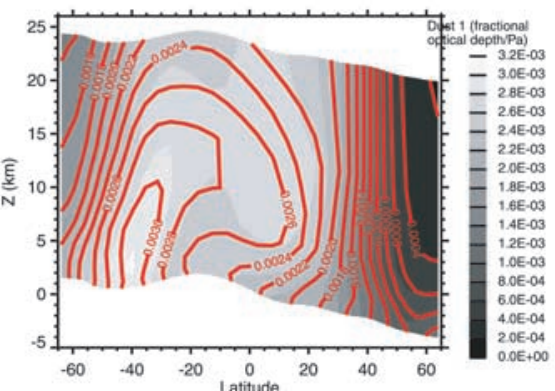

B

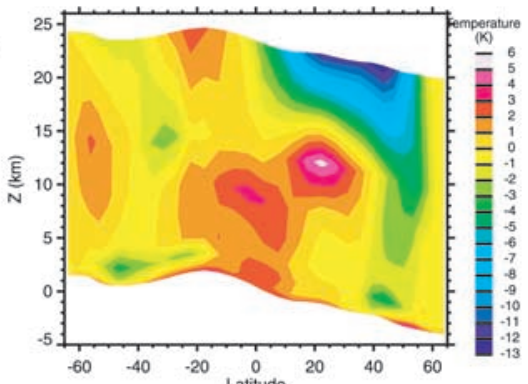

D
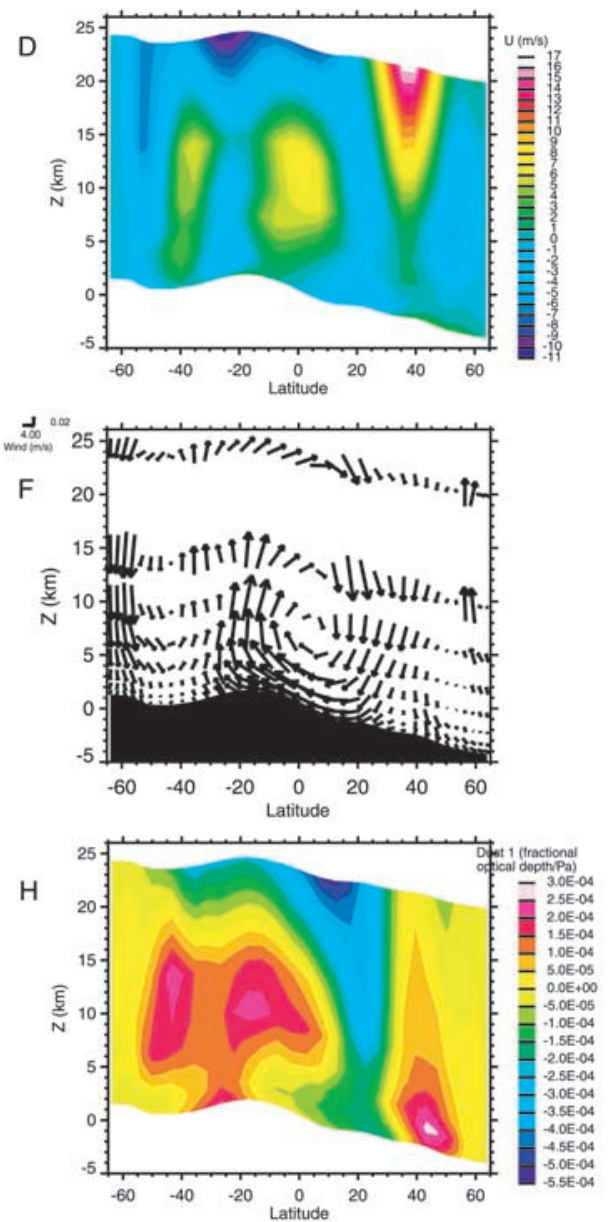

Figure 4. Same as Figure 2, except for $L_{s}=270$. 